\title{
Strategy-dependent effects of working-memory limitations on human perceptual decision-making
}

\author{
Kyra Schapiro $^{1}$, Krešimir Josić ${ }^{2,3}$, Zachary P. Kilpatrick ${ }^{4,5}$, and Joshua I. Gold ${ }^{1}$
}

${ }^{1}$ Department of Neuroscience, University of Pennsylvania, Philadelphia, PA, USA

${ }^{2}$ Department of Mathematics, University of Houston, Houston, TX, USA

${ }^{3}$ Department of Biology and Biochemistry, University of Houston, Houston, TX, USA

${ }^{4}$ Department of Applied Mathematics, University of Colorado Boulder, Boulder, CO, USA

${ }^{5}$ Institute of Cognitive Science, University of Colorado Boulder, Boulder, CO, USA 


\section{Abstract}

Deliberative decisions based on an accumulation of evidence over time depend on working

3 memory, and working memory has limitations, but how these limitations affect deliberative decision-

4 making is not understood. We used human psychophysics to assess the impact of working-memory

5 limitations on the fidelity of a continuous decision variable. Participants decided the average location of

6 multiple visual targets. This computed, continuous decision variable degraded with time and capacity in

7 a manner that depended critically on the strategy used to form the decision variable. This dependence

8 reflected whether the decision variable was computed either: 1) immediately upon observing the

9 evidence, and thus stored as a single value in memory; or 2) at the time of the report, and thus stored as

10 multiple values in memory. These results provide important constraints on how the brain computes and

11 maintains temporally dynamic decision variables. 


\section{Introduction}

Many perceptual, memory-based, and reward-based decisions depend on an accumulation of

14 evidence over time (Brody \& Hanks, 2016; Gold \& Shadlen, 2007; Ratcliff et al., 2016; Shadlen \&

15 Shohamy, 2016; Summerfield \& Tsetsos, 2012). This dynamic process, which can operate on timescales

16 ranging from tens to hundreds of milliseconds for many perceptual decisions to seconds or longer for

17 reward-based decisions (Bernacchia et al., 2011; Gold \& Stocker, 2017), depends on working memory

18 to maintain representations of new, incoming evidence and/or the aggregated, updating decision variable.

19 Working memory is known to be constrained by capacity and temporal limitations (Bastos et al., 2018;

20 Cowan et al., 2008; Funahashi et al., 1989; Oberauer et al., 2016; Panichello et al., 2019; Ploner et al.,

21 1998; Schneegans \& Bays, 2018; White et al., 1994), which implies such limitations may also constrain

22 decision performance when the decision requires information to be maintained in working memory.

23 Several previous studies failed to identify such constraints on working-memory-dependent decisions but

24 used tasks involving binary choices, which may have a low sensitivity to known working-memory

25 limitations (Liu et al., 2015; Waskom \& Kiani, 2018). It remains unclear how working-memory

26 limitations affect decisions that require interpreting and storing continuously valued quantities,

27 representations of which are known to degrade in working-memory (Ploner et al., 1998; Schneegans \&

28 Bays, 2018; Wei et al., 2012; White et al., 1994).

To assess such effects, we examined the relationship between decision-making and working memory in the context of visuo-spatial tasks about continuous variables (visual target locations) that are

31 sensitive to capacity and temporal limitations of working memory (Bastos et al., 2018; Funahashi et al.,

32 1989; Panichello et al., 2019; Ploner et al., 1998; Schneegans \& Bays, 2018; White et al., 1994).

33 Specifically, we required human participants to indicate a spatial location that was informed by one or

34 more briefly presented visual stimuli (“disks"; Fig. 1) after a variable delay. We compared the effects of

35 variable set size and delay when the remembered location corresponded to either: 1) the perceived 
36 location (angle) of a specific disk, identified at the time of interrogation (comparable to prior studies

37 (Ploner et al., 1998; Schneegans \& Bays, 2018; Wei et al., 2012; White et al., 1994)); or 2) the computed

38 mean angle of a set of multiple disks, which has not been examined in detail. Additionally, we examined

39 the effects of working-memory limitations on computed locations under two conditions that are

40 representative of certain decision-making tasks. The first was a "simultaneous" condition in which all

41 disks (and thus all information) were presented at once. The second was a "sequential" condition in which

42 one disk was presented later than the others. This condition required participants to adjust to a within-

43 trial change of available decision-relevant information, typifying decisions that require evidence

44 accumulation over time.

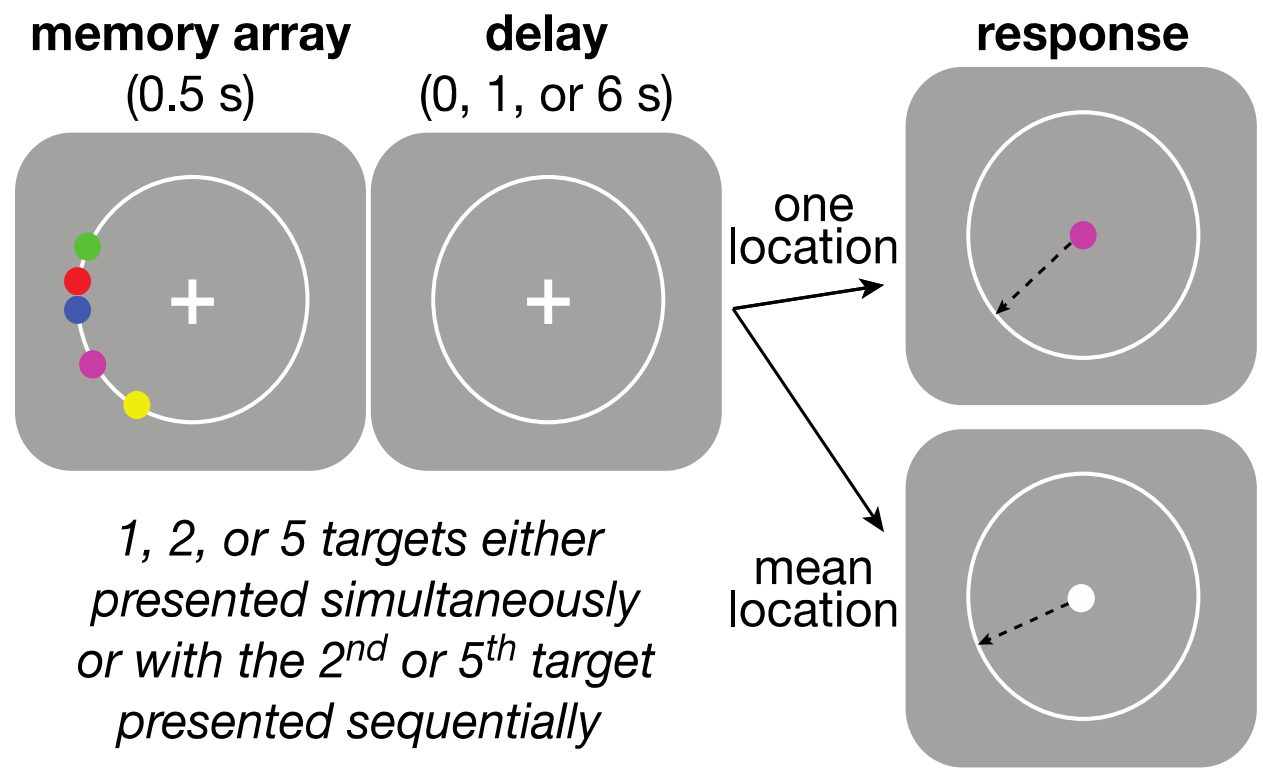

Figure 1: Behavioral task. Participants were asked to maintain visual fixation on the center cross while an array of colored disks was presented for $0.5 \mathrm{~s}$, followed by a variable delay and finally the presentation of a visual cue that had a color that was either: 1) the same as one of the disks, indicating that the participant should use the mouse to mark the remembered location of that disk ("perceptual" trial) or 2) white, indicating that the participant should mark the mean angle of the array ("computed" trial). Perceptual and Computed trials were separated by blocks. Participants knew in advance which block they were performing, but not which disk would be probed on any given trial, during Perceptual blocks. The number of disks and length of the delay period were varied randomly within each block. Blocks were also defined by the temporal presentation of the disks. In "simultaneous" blocks all disks were presented at once, whereas in "sequential" blocks, the final disk (most counterclockwise) was presented midway through the variable delay. 
For spatial working-memory tasks, the precision of working memory for perceived spatial

46 locations is often well described by diffusion dynamics (Compte, 2000; Kilpatrick, 2018; Kilpatrick et

47 al., 2013; Laing \& Chow, 2001) that are commonly implemented in "bump-attractor" models of working

48 memory (Compte, 2000; Constantinidis et al., 2018; Laing \& Chow, 2001; Riley \& Constantinidis, 2015;

49 Wei et al., 2012; Wimmer et al., 2014) (Fig. 2a). Our analyses built on this framework by examining

50 memory diffusion dynamics for the different task conditions and potential decision strategies. For the

51 conditions we tested, most participants behavior was well fit by one of two strategies, each with its own

52 constraints on decision performance based on different working-memory demands. The first strategy was

53 to compute the decision variable (mean disk angle) immediately upon observing the evidence (individual

54 disk angles), and then store that value in working memory in a manner that, like for the memory of a

55 single perceived angle, could be modeled as a single particle with a particular diffusion constant

56 (Average-then-Diffuse model; AtD; Fig. 2b parallel purple and solid black lines). The second strategy

57 was to maintain the representations of all disk locations in working memory, modeled as separate

58 diffusing particles, and then to combine them into a decision variable only at the time of the decision

59 (Diffuse-then-Average model; DtA). Such strategy use results in a diffusion constant for the average that

60 is inversely related to the number of points (Fig. 2b; magenta and dashed black lines). These two

61 strategies had slightly different predictions and formulations when samples were presented sequentially

62 (Fig. 2c,d). Our results show that like perceived angles, memory for computed mean angles degraded

63 with increased set size (of relevant information) and delay between presentation and report. However,

64 the degree of degradation depended strongly on the strategy used to compute the decision variables,

65 implying that multiple, strategy- and task-dependent effects of working-memory should be considered in

66 the construction of future neural and computational models of decision-making. 

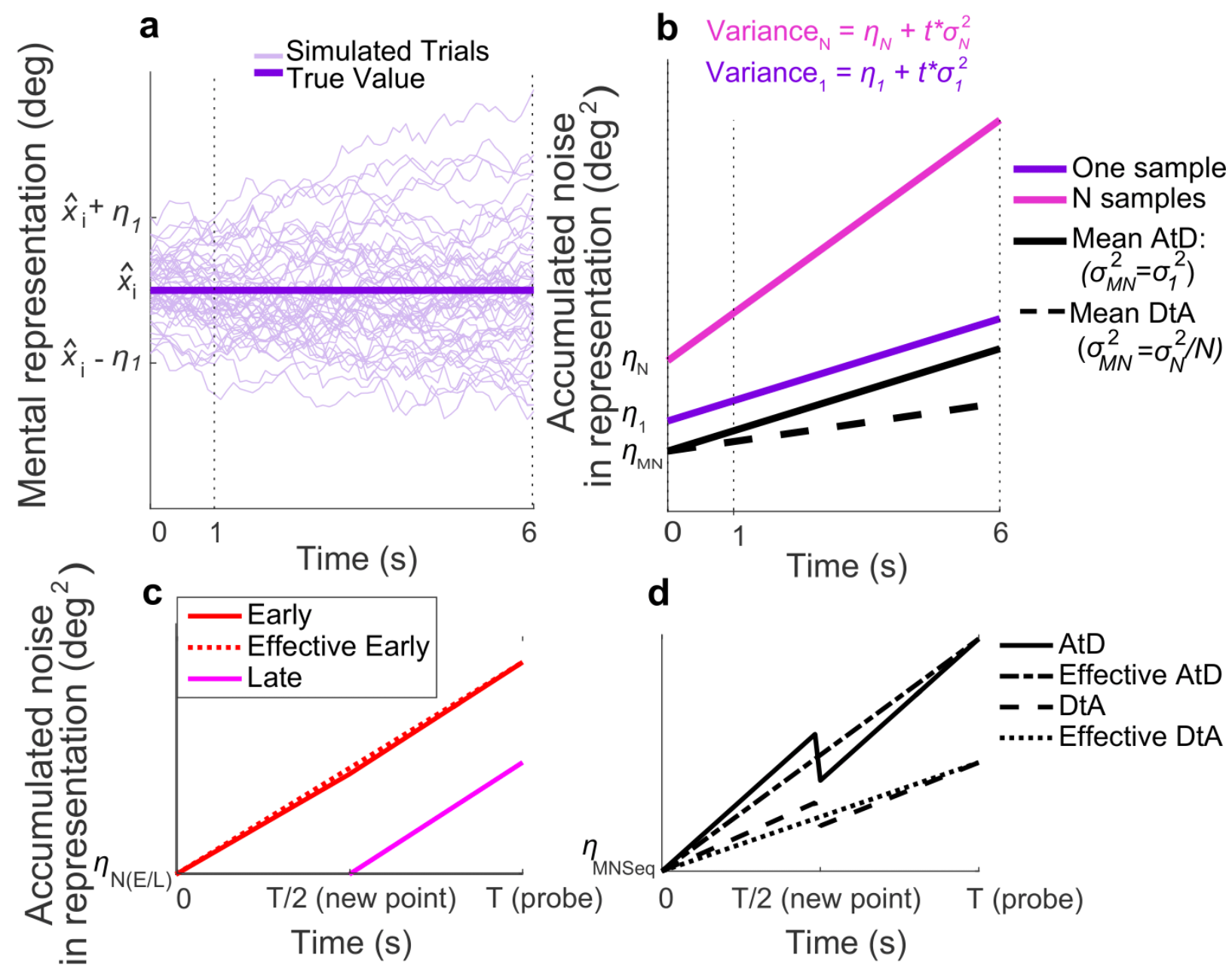

Figure 2: Diffusion model and predictions for different strategies. a) 50 simulated trials of the modeled representation of a single memorandum, $\hat{x}_{\mathrm{i}}$, experiencing Brownian diffusion. At time $t$, the report, $r_{t}, l$, is the location of the particle (motor noise is included in $\eta_{1}$ ). Note the increase in variance over time, which corresponds to decreased memory precision. b) Linear accumulation of noise (variance) for single or multiple perceived items (colors as indicated) or computed mean values using two different strategies. In each case, the memory representation starts with some initial, additive error, $\eta_{N}$, and diffuses over time with a diffusion constant $\sigma_{N}^{2}$, where $\mathrm{N}$ indicates set size. For the Average-then-Diffuse (AtD) model, the average over the presented stimuli is calculated immediately, and this single value is stored. Thus, the diffusion constant is identical between a Perceived and a Computed item (parallel purple and black lines), though the encoding may be different (i.e., the initial errors, $\eta_{l}$ and $\eta_{M N}$, may not be equal). For the Diffuse-then-Average (DtA) model, all items are stored until the probe time. Thus, the diffusion constant of the Computed item is $1 / N^{\text {th }}$ the diffusion constant of the multiple Perceived items held in memory. c) Accumulation of noise for Perceived items presented sequentially. Note that when the new point is added at time $T / 2$, the diffusion constant for previously presented items (Early) changes slightly because of the increased load. The "effective Early" trace shows the net gain in variance over time that would be expected when sampling the error only at time $T$, as was done in this study. d) Accumulation of noise for Computed items under sequential presentation conditions for both models. At time=T/2, the final point is averaged, causing a change in the diffusion coefficients. The effective lines represent the measured change in variance over time one would measure when recording only at time T, as we did. In these examples $N=5, A=0.5$. 


\section{Results}

68 We measured the ability of human participants to remember spatial angles as a function of set

69 size (1, 2, or 5 items), delay duration $(0,1$, or 6 s), and task context (Perceived or Computed blocks). We

70 measured error between reported and probed angles as a proxy for working memory-representations and

71 inferred rates of memory degradation (diffusion constants) from the increase in variance of these errors

72 over time. Below we first describe results from Simultaneous conditions, in which all items were

73 presented simultaneously at the beginning of each trial, and show how capacity and temporal constraints

74 on working memory relate to the accuracy of computed decision variables. We then describe our findings

75 from Sequential conditions, in which one item was presented after the others in each trial, and show how

76 capacity and temporal constraints affect the process of evidence integration over time.

77 Simultaneous condition behavior

The difference in reports of Perceived spatial angles and the true probed location (i.e., the

response error) tended to be relatively unbiased in that the mean error across participants was not

80 significantly different from zero (Fig. 3a, full distributions in Fig. S1, individual participant mean errors

81 in Fig. S3-4). However, the variance of these errors increased roughly linearly over time (Fig. 3c),

82 consistent with predictions of particle diffusion models (Compte, 2000; Kilpatrick, 2018; Kilpatrick et

83 al., 2013; Laing \& Chow, 2001). This error variance also depended systematically on set size (Fig. 3c).

84 However the change in error variance over time (slope of variance increase) did not depend on set size

85 (ANOVA, significant effect of set size, $\mathrm{F}(2,32)=83.87, \mathrm{p}=1.88 \mathrm{e}-13$, and delay, $\mathrm{F}(2,32)=29.55$, $\mathrm{p}=5.37 \mathrm{e}-$

8608 , but no significant interaction between set size and delay, $F(4,64)=1.36, p=0.256)$.

Errors in reports of Computed (i.e., inferred mean) spatial angles relative to true mean angles

showed similar trends, albeit with a much weaker dependence on the number of items. Specifically,

89 Computed angle reports were also unbiased (mean error from the true value was not significantly

90 different from zero; Fig. 3b, S1,S3-4) but degraded (became more variable) with a roughly linear increase 
91 in variance over time (Fig. 3d). Error variance was higher at higher set sizes (set size 5 had higher

92 variances), but the rate of degradation in accuracy did not depend on set size (ANOVA, significant effect

93 of set size, $F(2,32)=13.53 p=5.515 \mathrm{e}-5$, and delay, $F(2,32)=130.79, p=4.441 \mathrm{e}-16$, but not their interaction,

$94 F(4,64)=0.538, p=0.708)$.
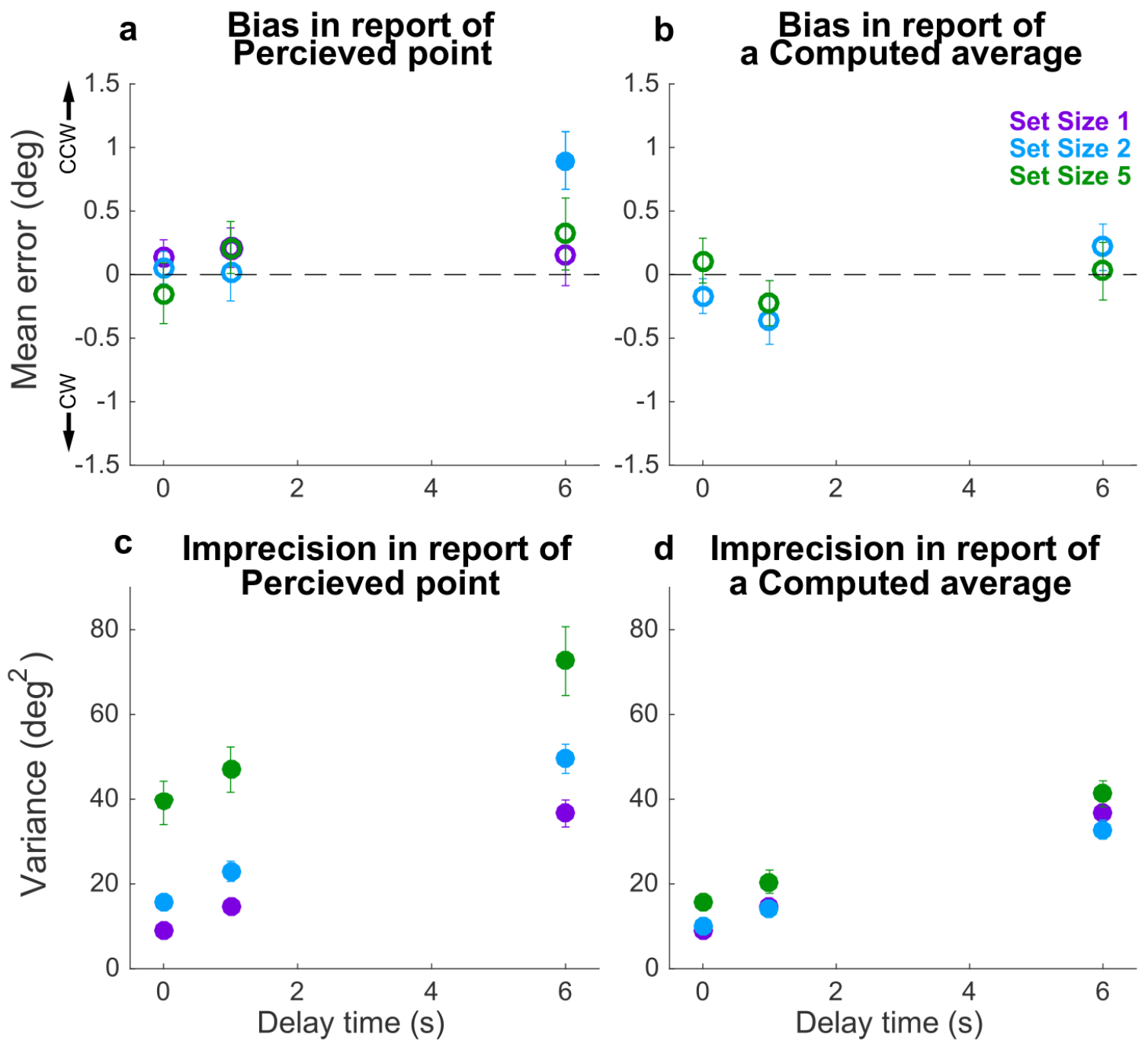

Figure 3: Behavioral summary for the Simultaneous condition. A) Mean Perceptual error for different set sizes (colors, as indicated) and delay time (abscissa). Filled points indicate two-tailed $t$-test for $H_{0}$ : mean=0, $p<0.05$. B) Mean Computed (inferred mean) error for different set sizes (colors, as indicated) and delay time (abscissa); for all tests mean error was not significantly different from zero (open circles). C) Variance in Perceptual errors, plotted as in A. D) Variance in Computed (mean) errors, plotted as in B. In each panel, points and error bars are mean \pm SEM across participants. 


\section{Simultaneous condition model fits}

To better understand the effects of delay and set size on working-memory representations of

97 Perceived and Computed angles for individual participants, we fit the AtD and DtA models (see Methods

98 for details) separately to data from each condition and participant (Table 1; the two models each had the

99 same number of free parameters and thus were compared using the log-likelihoods of the fits). Both models include terms that quantify separately the effect of set size on non-time-dependent noise (i.e., the variance in report errors with no delay; $\eta$ ) and the diffusion constant (i.e., the rate at which the variance

102 of the errors increases over time for a single Perceived point; $\sigma_{1}^{2}$ ). The $A$ parameter governs the

103 relationship between $\sigma_{1}^{2}$ and the diffusion constant for multiple Perceived points $\left(\sigma_{N}^{2}\right)$ (i.e., $\sigma_{N}^{2}=\sigma_{1}^{2 *} N^{A}$ ).

\begin{tabular}{|l|l|l|l|l|l|l|l|}
\hline & $\begin{array}{l}\text { Set } \\
\text { size } \\
(\boldsymbol{N})\end{array}$ & $\begin{array}{l}\text { Number } \\
\text { best-fit } \\
\text { participants }\end{array}$ & $\eta_{\boldsymbol{I}}$ & $\eta_{N}$ & $\eta_{M N}$ & $\sigma_{I}{ }^{2}$ & $A$ \\
\hline AtD & 2 & 8 & $10.79 \pm 1.45$ & $16.49 \pm 2.67$ & $9.39 \pm 1.07$ & $4.85 \pm 0.44$ & $0.0892 \pm 0.24$ \\
\hline & 5 & 14 & $9.80 \pm 1.18$ & $36.88 \pm 5.33$ & $14.79 \pm 1.50$ & $4.34 \pm 0.47$ & $0.0051 \pm 0.07$ \\
\hline DtA & 2 & 9 & $8.22 \pm 1.35$ & $14.16 \pm 3.13$ & $10.45 \pm 2.09$ & $3.67 \pm 0.58$ & $0.61 \pm 0.22$ \\
\hline & 5 & 3 & $7.63 \pm 0.30$ & $45.49 \pm 17.03$ & $21.10 \pm 7.59$ & $5.14 \pm 0.28$ & $0.49 \pm 0.14$ \\
\hline
\end{tabular}

Table 1. Summary of model fits for the Simultaneous condition. Parameters are: 1) $\eta_{1}$, non-timedependent noise of a single value; 2) $\boldsymbol{\eta}_{N}$, non-time-dependent noise of $N$ points; 3) $\boldsymbol{\eta}_{\boldsymbol{M} N}$, non-timedependent noise of the mean of $N$ points; 4) $\boldsymbol{\sigma}_{1}{ }^{2}$, diffusion constant of a single point; and 5) $\boldsymbol{A}$, diffusion cost of additional points. For each parameter, the maximum likelihood estimates (mean over participants \pm SEM) are given for the participants best fit with a particular model.

For the participants best fit by the AtD model, the mean, best-fitting values of $A$ were close to zero,

105 which reflects the lack of interaction between set size and delay seen in the Perceptual ANOVA in Fig. 3c

106 (because in this model, $\sigma_{N}^{2}=\sigma_{1}^{2}$ when $A=0$ ). Conversely, for the participants best fit by the DtA model,

107 the mean, best-fitting values of $A$ were slightly higher, which reflects the lack of interaction in the

108 Computed ANOVA in Fig. 3d (because in this model, the diffusion of a Computed point scales with $\sigma_{N}^{2}$,

109 specifically $\sigma_{M N}^{2}=\sigma_{N}^{2} / \mathrm{N}$; thus, $\sigma_{M N}^{2}$ does not differ from $\sigma_{l}^{2}$ in DtA only when $\sigma_{N}^{2}>\sigma_{l}^{2}$, which occurs

110 when $A>0$ ). Of note, when $A=1$, the AtD and DtA models make identical predictions, namely $\sigma_{M N}{ }^{2}=\sigma_{1}^{2}=$ 
$111 \sigma_{1}^{2 *} N^{A} / N=\hat{\sigma}_{N}^{2} / N$. Across the population, the $95 \%$ confidence intervals for $A$ (as determined by the SEM

$112 A$ values across the population) did not overlap with 1, supporting the distinguishability of the two models

113 on average participants (although not for each individual participant; Fig. S7).

114 Simultaneous condition model validation

115 When $A$ differs from one, AtD and DtA make distinct, strong assumptions about the diffusion

116 constant relationships between either single (AtD) or multiple (DtA) Perceived angles(s) versus a

117 Computed average angle, as depicted in Fig. 2b. We used these assumptions to validate whether the

118 better-fitting model and best-fit parameters for a given participant at a given set size were likely to

119 produce the participant's behavior. Specifically, the AtD model assumes that the diffusion constant for a

120 single Perceived angle and for a Computed average angle are the same because both involve the memory

121 of a single value (eq. 9). In contrast, the DtA model assumes that the diffusion constant for a Computed

122 average angle is $1 / N^{\text {th }}$ the diffusion constant for $N$ points because all $N$ points are held in memory prior

123 to averaging (eq.10). We analyzed how consistent these assumptions were with the behavioral data (Fig.

124 4). Specifically, for each participant we fit a line to the measured error variances as a function of delay

125 for a given set size in both Perceived and Computed blocks to estimate the change in variance over time

126 (the empirical diffusion constant estimates: $\hat{\sigma}_{I}^{2}, \hat{\sigma}_{N}^{2}, \hat{\sigma}_{M N}{ }^{2}$, where $N=2$ or 5 for the two set sizes). We then

127 compared the differences of these empirical estimates to the differences predicted between diffusion

128 constants by the best fit model for a given participant.

In general, the participant data conformed to the model predictions of the best-fit model for that

130 participant, despite substantial individual variability. For participants whose data were best fit by the AtD

131 model, empirical estimates of the diffusion constant $\left(\hat{\sigma}_{M N}^{2}\right)$ from Computed blocks tended to be similar

132 to the empirical estimates of the diffusion constant for a single Perceptual point $\left(\hat{\sigma}_{1}^{2}\right.$; Fig. 4a,c). 


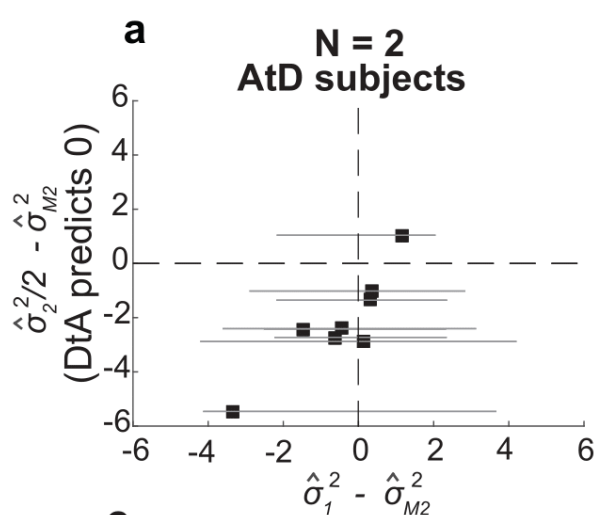

C

$\mathbf{N}=\mathbf{5}$

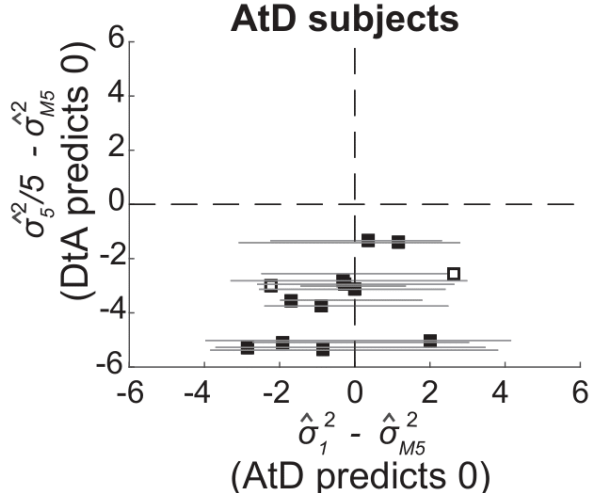

(AtD predicts 0$)$

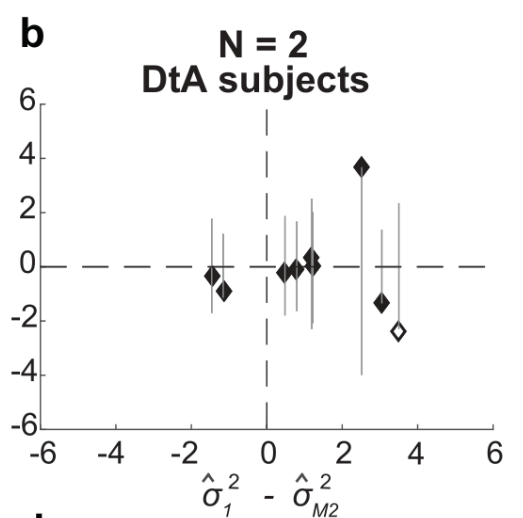

d $\quad \mathrm{N}=5$

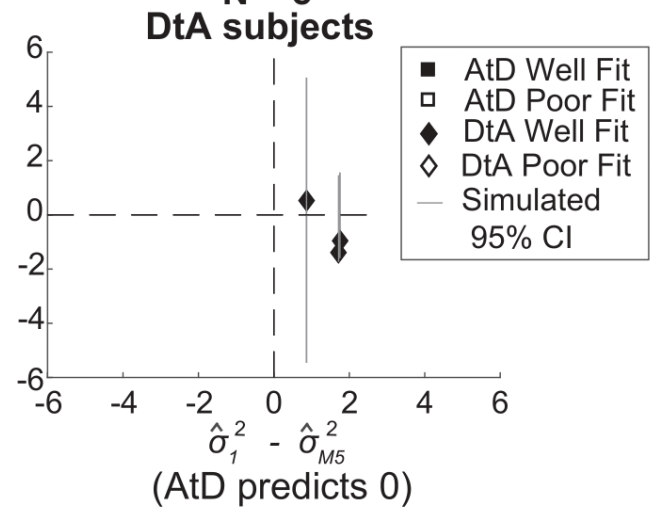

Figure 4: Comparisons of empirical and model-based diffusion constant relationships for the Simultaneous condition. In each panel, the abscissa shows the difference between: 1) empirical estimates of the diffusion constant for a Computed value measured by fitting a line to measured variance as a function of delay time for set size $2\left(\hat{\sigma}_{M 2}{ }^{2}, \mathbf{a}, \mathbf{b}\right)$ or $5\left(\hat{\sigma}_{M 2}{ }^{2}, \mathbf{c}, \mathbf{d}\right)$, and 2) the empirical estimates of the diffusion constant for a single Perceived value $\left(\hat{\sigma}_{1}{ }^{2}\right)$. The Average-then-Diffuse (AtD) model predicts a difference of zero. The ordinate shows the difference between: 1) the empirical estimate of Computed diffusion constants $\hat{\sigma}_{M 2}{ }^{2}$ or $\hat{\sigma}_{M 5}{ }^{2}$, and 2) the empirical estimates of the diffusion constant for multiple Perceived values $\left(\hat{\sigma}_{2}^{2}\right.$ or $\left.\hat{\sigma}_{5}{ }^{2}\right)$ divided by the number of points. The Diffuse-then-Average (DtA) model predicts a difference of zero. Each point was obtained using data from individual participants, separated by whether they were best fit by the AtD $(\mathbf{a}, \mathbf{c})$ or DtA $(\mathbf{b}, \mathbf{d})$ model for the given set-size condition. Lines represent $95 \%$ confidence intervals computed by simulating data using the best-fit parameters for the given fit and repeating the empirical estimate comparison procedure. Closed symbols indicate participants who fell within the $95 \%$ confidence interval for their best-fit model.

133 Specifically, for all but two participants, the empirical diffusion constant differences fell within the $95 \%$

134 confidence interval of simulated distribution. Likewise, for participants whose data were best fit by the

135 DtA model, empirical estimates of the diffusion constant $\left(\hat{\sigma}_{M N}{ }^{2}\right)$ from Computed blocks tended to be

136 similar to the empirical estimates of the diffusion constant for multiple Perceptual points divided by the

137 set size $\left(\hat{\sigma}_{N}{ }^{2} / N ;\right.$ Fig. $\left.4 \mathrm{~b}, \mathrm{~d}\right)$. Specifically, for all but one participant, empirical diffusion constant differences

138 fell within the $95 \%$ confidence interval of the simulated distribution. For some participants, the diffusion 
constant relationship conformed to the expectations of both models (point lying near origin in Fig. 4).

140 These analyses thus support the idea that for most subjects, their behavior was well captured by their

141 better-fitting model.

142 Summaries of the predicted report-error variances by the AtD and DtA fits for well-fit participants

143 are shown in Fig. 5. Overall, the model predictions match participant behavior. In general, AtD

144 participant behavior was predicted by diffusion constants that were the same for either one Perceived

145 location or the mean Computed location based on 2 or 5 points (i.e., parallel lines in Fig. 5e,g). DtA

146 participant behavior was well predicted by diffusion constants that were larger for multiple Perceived

147 points compared to Single Perceived points (Fig. 5f,h). As predicted by the DtA model, the Computed

148 point errors for DtA participants were well predicted by $1 / \mathrm{N}^{\text {th }}$ the diffusion constant for multiple

149 Perceived points (Fig. 5e-h).

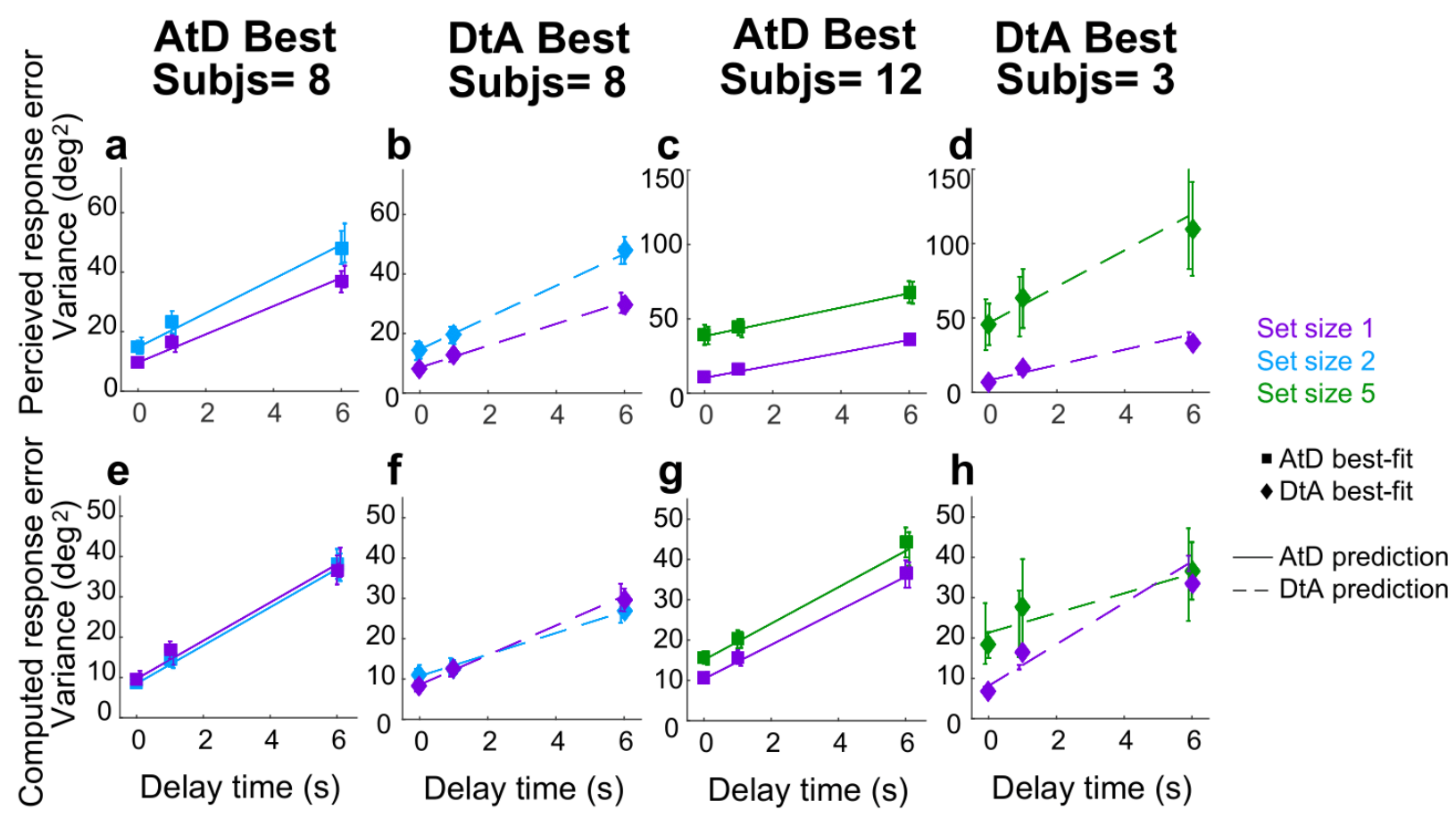

Figure 5. Comparison of model prediction to participant data for the Simultaneous condition. Each panel shows the empirical variance of participant errors (points and error bars are mean \pm SEM data across participants) and model predictions (lines, based on the mean best-fitting parameters across participants for the given model) for the participants best fit by the given model (AtD or DtA) for the given condition, as labeled above each column. A-D) Perceived blocks. E-F) Computed blocks. 
$\underline{\text { Simultaneous condition strategy comparisons }}$

152 for the two set-size conditions (Fig. 6). Specifically, equal numbers of well-fit participants were best fit

153 by the $\operatorname{AtD}(n=8)$ and the DtA $(n=8)$ model for a set size of 2, and as such neither model was significantly

154 more likely to be a better fit (Wilcoxon signed-rank two-sided test for the median difference in the log-

155 likelihoods of fits of the two models to data from each participant $=0, p=0.756)$. In contrast, at set size 5 ,

156 the well-fit participants were more likely to be better fit by the AtD $(n=12)$ than the DtA $(n=3)$ model

157 ( $p=0.0027)$. Participants who were not poorly fit at either set size were more likely to be better fit by AtD

158 in set size 5 compared to set size 2 (Wilcoxon signed-rank two-sided test for equal median log-likelihoods

159 difference of fits of the two models across set sizes, $p=0.029)$. Additionally, the log likelihood difference

160 of strategy use did not correlate with the age of the participants (Pearson correlation, Fig. S8a, $p>0.20$ ).

161 These findings suggest that working-memory load may affect people's decision strategies, such that a

162 higher load seems to correspond to an increased tendency to discard information about individual samples

163 (disk locations) and hold only the relevant computed decision variable in memory.

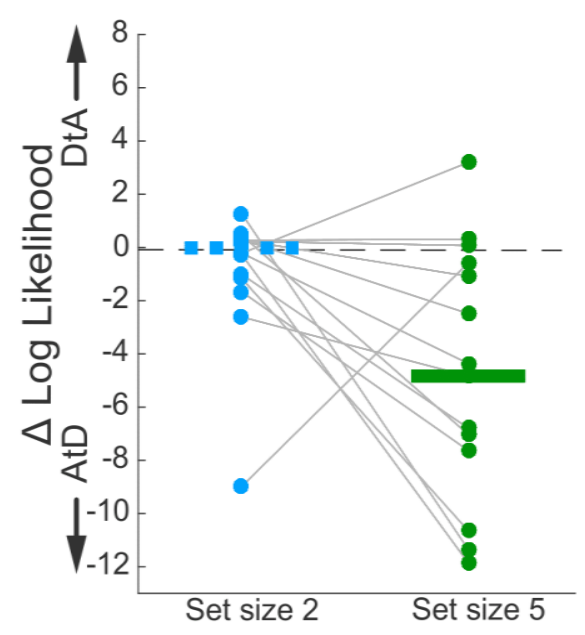

Figure 6. Difference in log likelihood between AtD and DtA fits for the Simultaneous condition. Negative values favor AtD. Each point represents the difference in fit log likelihoods for one participant; horizontal bars are medians (solid bar for set size 5 indicates two-sidesd Wilcoxon signedrank test for $H_{0}$ : median=0, $p=0.0027$ ). Positive values favor DtA, whereas negative favor AtD. Grey lines connect data generated by the same participant. Only participants whose data were well matched to one of the two models (i.e., within the $95 \%$ confidence intervals depicted in Fig. 4) were included. 
$\underline{\text { Sequential condition behavior }}$

We separately analyzed errors for Perceived reports of disks presented at the beginning (Early) or middle (Late) of a trial. Early Perceived reports tended to be relatively unbiased (mean error not significantly different from zero; Fig. 7a, full distributions in Fig. S2) but became more variable over time in a roughly linear manner (Fig. 7d), consistent with the predictions of the particle diffusion model. For higher set sizes, errors were more variable than at lower set sizes. The rate of variance increase over time did not depend on set size (ANOVA, significant effect of set size, $F(2,32)=33.44, p=1.45 \mathrm{e}-08$, and delay, $F(1,16)=77.02, p=1.64 \mathrm{e}-07$, but not their interaction, $F(2,32)=0.15, p=0.256)$. Late Perceived reports were likewise unbiased (mean error not significantly different from zero; Fig. 7b, full distributions

173 in Fig. S2) and degraded in precision (i.e., increased in variance) over time (Fig.7e). However, this degradation did not depend on set size (ANOVA, significant effect of delay, $F(1,16)=39.28, p=1.12 \mathrm{e}-05$, but not set size, $F(1,16)=0.90, p=0.36$ or their interaction, $F(1,16)=0.0029, p=0.96)$.

points tended to be slightly biased towards the Early points for set size 2 (student two-sided $t$-test, $p<0.001)$ but not set size 5 ( $p>0.5$; Fig. $6 c$, full distributions in Fig. S2). The Computed report errors also increased in variance over time (Fig. 7f). The overall magnitude of this imprecision and its change over time depended systematically on the number of items to remember, such that more items corresponded to a slightly greater overall variance in reports at short delays, but less gain in variance over time 182 (ANOVA, significant effect of set size, $F(2,32)=7.73 p=1.8 \mathrm{e}-3$, delay, $F(1,16)=73.76, p=2.18 \mathrm{e}-07$, and 183 their interaction, $F(2,32)=6.81, p=3.4 \mathrm{e}-3)$. This interaction of delay and set size suggests the

184 representation of the Computed value diffused in working memory with a different diffusion constant 185 than for a single Perceived value. This interaction is consistent with predictions of both the AtD and DtA 186 models under these conditions, though the nature of this interaction depends on the specific model, as detailed below. 

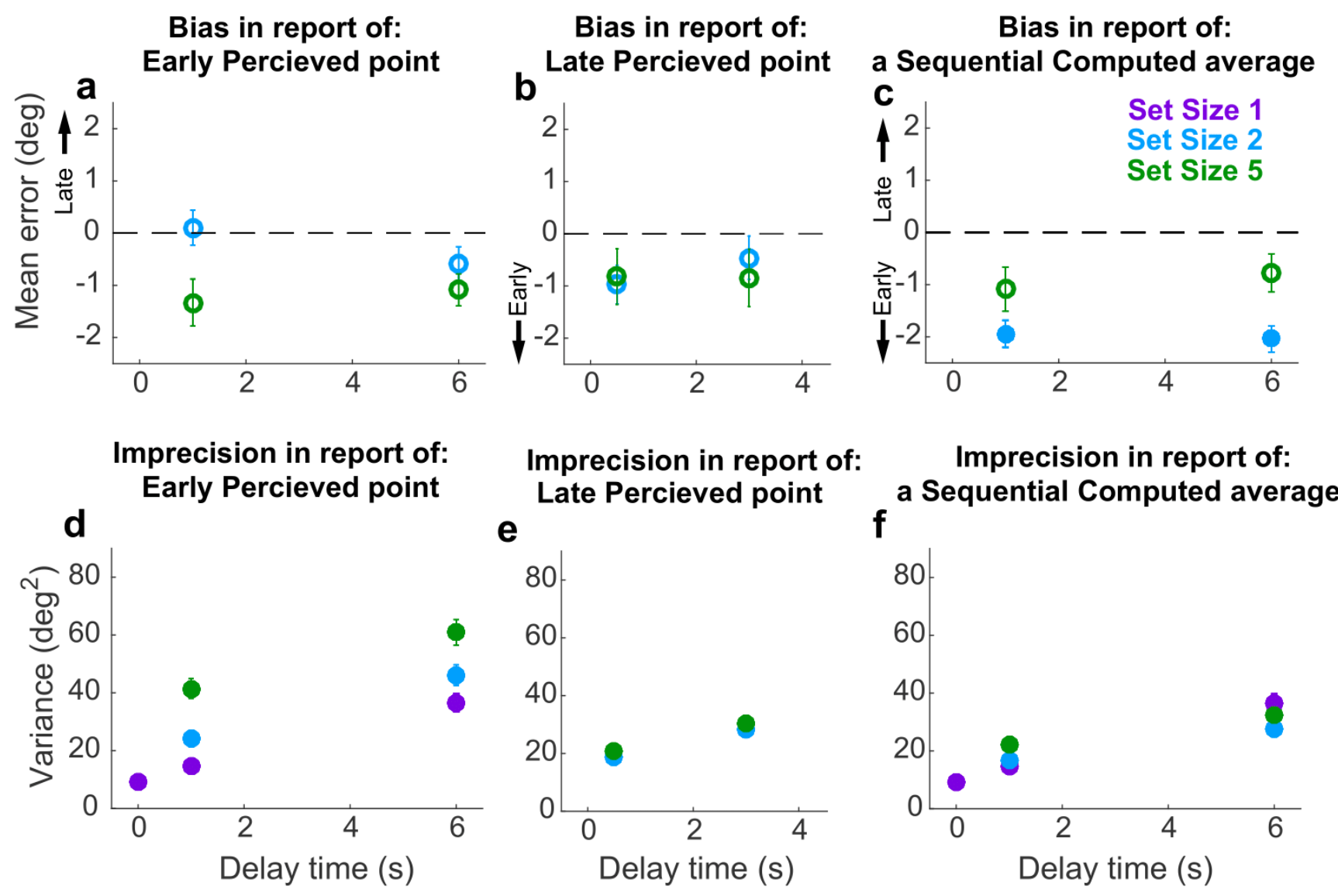

Figure 7: Behavioral summary for the Sequential condition. a) Mean error for initially presented (Early) Perceptual points for different set sizes (colors, as indicated) and delay time (abscissa). b) Mean error for midway presented (Late) Perceptual points for different set sizes (colors, as indicated) and delay time (abscissa). c) Mean Computed (inferred mean) error for different set sizes (colors, as indicated) and delay time (abscissa). Filled points in a-c indicate two-tailed student $t$-test for $H_{0}$ : mean $=0, p<0.05$. d) Variance in Early Perceptual errors plotted as in a. e) Variance in Late Perceptual errors, plotted as in b. f) Variance in Computed (mean) errors, plotted as in c. In each panel, points and error bars are mean \pm SEM across participants.

To better understand the effects of delay and set size on working-memory representations of

190 Perceived and Computed locations for individual participants under Sequential conditions, we fit the AtD

191 and DtA models separately to data from each condition and participant (Table 2; the two models each

192 had the same number of free parameters and thus were compared using the log-likelihoods of the fits).

193 Recall that the $\eta$ parameters quantify the effect of set size on non-time-dependent noise (noise when delay

194 is zero), whereas $\sigma_{l}^{2}$ is the model-based estimate of the diffusion constant for a single Perceived point. 


\begin{tabular}{|l|l|l|l|l|l|l|l|l|}
\hline & $\begin{array}{l}\text { Set } \\
\text { size } \\
(\boldsymbol{N})\end{array}$ & $\begin{array}{l}\text { Number } \\
\text { best-fit } \\
\text { participants }\end{array}$ & $\eta_{1}$ & $\eta_{N E}$ & $\eta_{N L}$ & $\eta_{M N-s e q}$ & $\sigma_{1}^{2}$ & $A$ \\
\hline AtD & 2 & 9 & $9.44 \pm 1.47$ & $20.32 \pm 3.89$ & $16.18 \pm 3.16$ & $14.02 \pm 1.58$ & $4.44 \pm 0.73$ & $-0.34 \pm 0.44$ \\
\hline & 5 & 9 & $10.69 \pm 1.21$ & $37.06 \pm 5.22$ & $13.19 \pm 2.06$ & $15.94 \pm 1.69$ & $4.22 \pm 0.73$ & $-0.09 \pm 0.15$ \\
\hline DtA & 2 & 8 & $10.25 \pm 1.53$ & $18.30 \pm 3.31$ & $17.43 \pm 1.88$ & $14.00 \pm 3.11$ & $4.58 \pm 0.52$ & $-3.00 \pm 2.58$ \\
\hline & 5 & 8 & $9.11 \pm 1.69$ & $36.59 \pm 5.54$ & $22.27 \pm 4.37$ & $24.45 \pm 4.60$ & $4.43 \pm 0.57$ & $-3.89 \pm 2.90$ \\
\hline
\end{tabular}

Table 2. Summary of model fits for the Sequential condition. Parameters are: 1) $\boldsymbol{\eta}_{\boldsymbol{1}}$, non-time-dependent noise of a single value; 2) $\boldsymbol{\eta}_{N E}$, non-time-dependent noise of the Early $N-1$ points; 3) $\boldsymbol{\eta}_{N L}$, non-timedependent noise of the Late $N^{\text {th }}$ points; 4) $\boldsymbol{\eta}_{M N \text {-seq }}$, non-time-dependent noise of the mean of $N$ points; 5) $\boldsymbol{\sigma}_{1}{ }^{2}$, diffusion constant of a single point; and 6) $\boldsymbol{A}$, diffusion cost of additional points. For each parameter, the maximum likelihood estimates (mean over participants \pm SEM) is given for the participants best fit with a particular model. ANOVA. Unlike in the Simultaneous Condition, the participants best fit by DtA had negative $A$ values at both set sizes, implying that the diffusion constant for multiple Perceived items became closer to zero as the number of points increased. While counterintuitive to the concept that adding more points should increase the diffusion constant, such a negative $A$ can be explained by ceiling effects: if a participant has high levels of non-time-dependent noise, they have less room to degrade while still accurately tracking

202 the target (i.e., not having a lapse). Alternatively, the presentation of a new point may have had a 203 stabilizing effect on the ensemble by creating directional drift towards the new point rather than random diffusion in the remaining points (Almeida et al., 2015; Wei et al., 2012), which is not inherently accounted for in any of the present models. As in the Simultaneous condition, the models make identical predictions when $A=1$. Across the population, 95\% confidence intervals of $A$ did not overlap with one, supporting the distinguishability of the two models; however, this difference from one was not always

208 true for individual participants (Estimates of $A$ on a participant-by-participant basis are shown in Fig. S56). 


\section{$\underline{\text { Sequential condition model validation }}$}

As in the Simultaneous condition, the Sequential condition models also make predictions about

212 the relationship between the diffusion constants of remembered Computed and Perceived values. Once

213 again, we assessed how well participant behavior matched these assumptions, detailed in eq. 11 for AtD

214 and eq. 12 for DtA (Fig. 8). We fit a line to the measured variances in reporting error as a function of

215 delay for a given set size in both Perceived and Computed Sequential blocks to estimate the change in

216 variance over time (the empirical diffusion constant estimates: $\hat{\sigma}_{1}^{2}, \hat{\sigma}_{N E}^{2}, \hat{\sigma}_{N L}^{2}, \hat{\sigma}_{M N-s e q}{ }^{2}$, where $N=2$ or 5 for

217 the two set sizes). We then compared the difference of these empirical estimates to the predictions of the

218 best-fit model for each participant (Fig. 8). We used our parametric bootstrapping variant to create

219 simulated distributions of expected deviation from the model-defined diffusion constant relationships for

220 each subject as described previously.

In general, the participant data conformed to the model predictions of the best-fit model for that

223 model ( $\mathrm{n}=9$ for both set sizes), empirical estimates of the diffusion constant $\left(\hat{\sigma}_{M N-s e q}{ }^{2}\right)$ from Computed

224 blocks tended to be similar to the expected fraction of the empirical estimates of the diffusion constant

225 for a single point (Fig. 8a,c). Specifically, for every participant the empirical diffusion constant differences fell within the $95 \%$ confidence interval computed from simulations using the model fits. For

227 participants whose data were best fit by the DtA model ( $\mathrm{n}=8$ for both set sizes), empirical estimates of

228 the diffusion constant $\left(\hat{\sigma}_{M N}{ }^{2}\right)$ from Computed blocks tended to be similar to the expected average of

229 empirical estimates of the diffusion constant for multiple points $\left(0.5 \hat{\sigma}_{N L}^{2}+(N-1)^{*} \hat{\sigma}_{N E^{2}}{ }^{2} / N^{2}\right.$; Fig. S10b,d).

230 Specifically, for 7 participants, empirical diffusion constant differences fell within the $95 \%$ confidence

231 interval computed from simulations using the model fits. The remaining subject was considered poorly

232 fit and not considered in further analyses. 

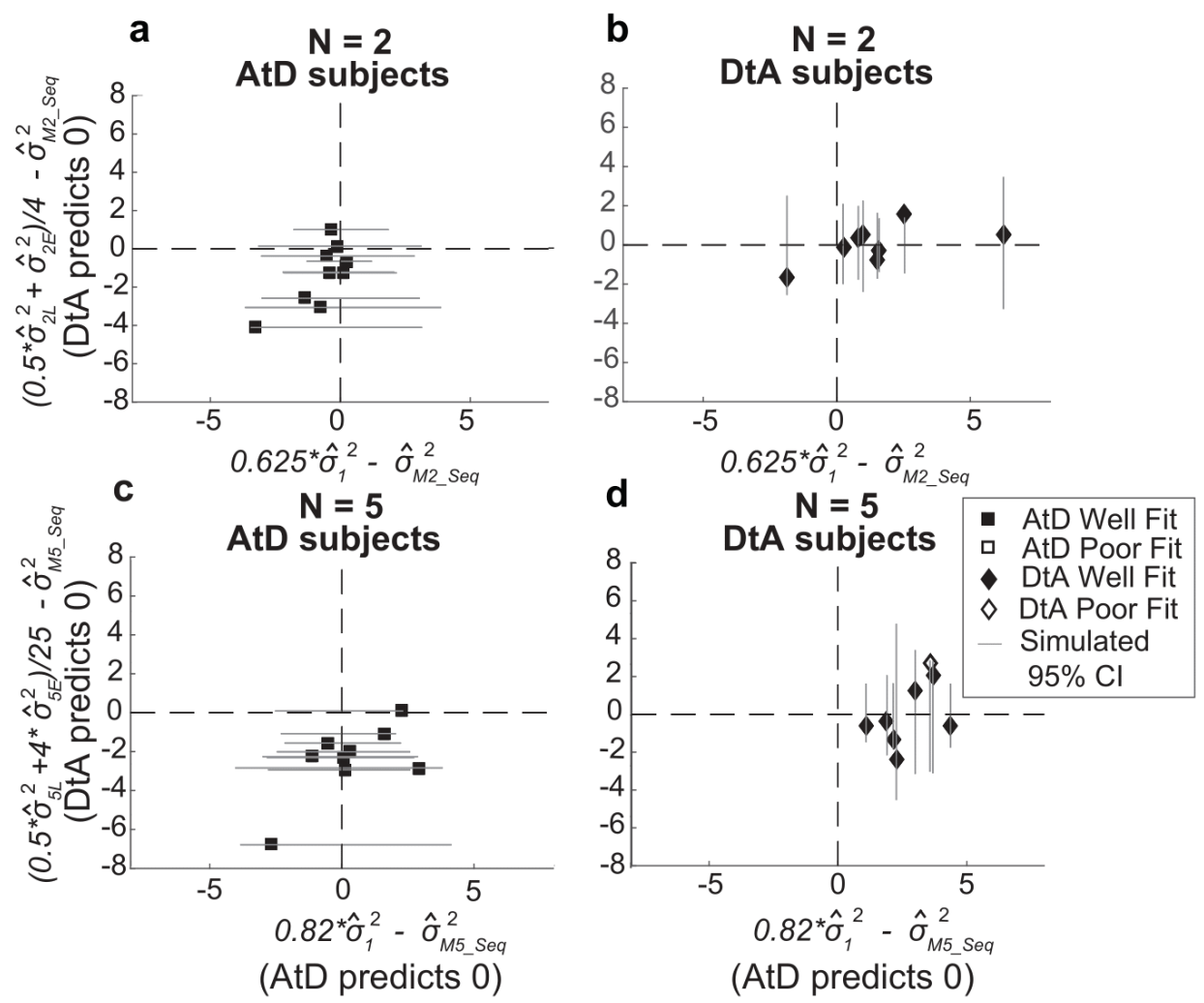

Figure 8: Comparisons of empirical and model-based diffusion constants. In each panel, the abscissa shows the difference between: 1) empirical estimates of the diffusion constant for a Computed value measured by fitting a line to measured variance as a function of delay time for set size $2\left(\hat{\sigma}_{M 2}{ }^{2}, \mathbf{a}, \mathbf{b}\right)$ or 5 $\left(\hat{\sigma}_{M 5}{ }^{2}, \mathbf{c}, \mathbf{d}\right)$, and 2) the empirical estimates of the diffusion constant for a single Perceived value $\left(\hat{\sigma}_{1}{ }^{2}\right)$ multiplied by the appropriate factor for the set size. The Average-then-Diffuse (AtD) model predicts a difference of zero. The ordinate shows the difference between: 1) the empirical estimate of Computed diffusion constants $\hat{\sigma}_{M 2}{ }^{2}$ or $\hat{\sigma}_{M 5}{ }^{2}$, and 2) the empirical estimates of the diffusion constant of a Computed value based on the DtA hypothesis. Therefore, a value of zero indicates a match between the DtA prediction of the Computed Diffusion constant and the empirically measured estimate. Points are data from individual participants, separated by whether they were best fit by the AtD (a,c) or DtA (b,d) model for the given set-size condition. Lines are $95 \%$ confidence intervals computed by simulating data using the best-fit parameters for the given fit and repeating the empirical estimate comparison procedure. Close symbols indicate participants who fell within the $95 \%$ confidence interval for their best-fit model.

234 In general, participants best fit by AtD on average exhibited diffusion constants that were lower for

235 Computed than Perceived values (Fig. 9i,k; lower slope of cyan/blue line versus purple line), with the

236 difference decreasing with increased set size, as expected due to averaging process (Fig. 2d). Additionally

237 for the participants best fit by AtD, both the Early and Late variances were on average fairly well matched

238 by their model predictions as well (Fig. 9a,e,c,g). Conversely, participants best fit by DtA exhibited 
239 diffusion constants that were notably smaller for Computed mean locations versus single Perceived

240 locations (Fig. 9j,1; lower slope of cyan/blue line versus purple line). The corresponding average

241 predictions by the best fit DtA models for error variance of Early and Late Points also aligned with

242 participant data from DtA fit participants (Fig. 9 b,f,d,h).

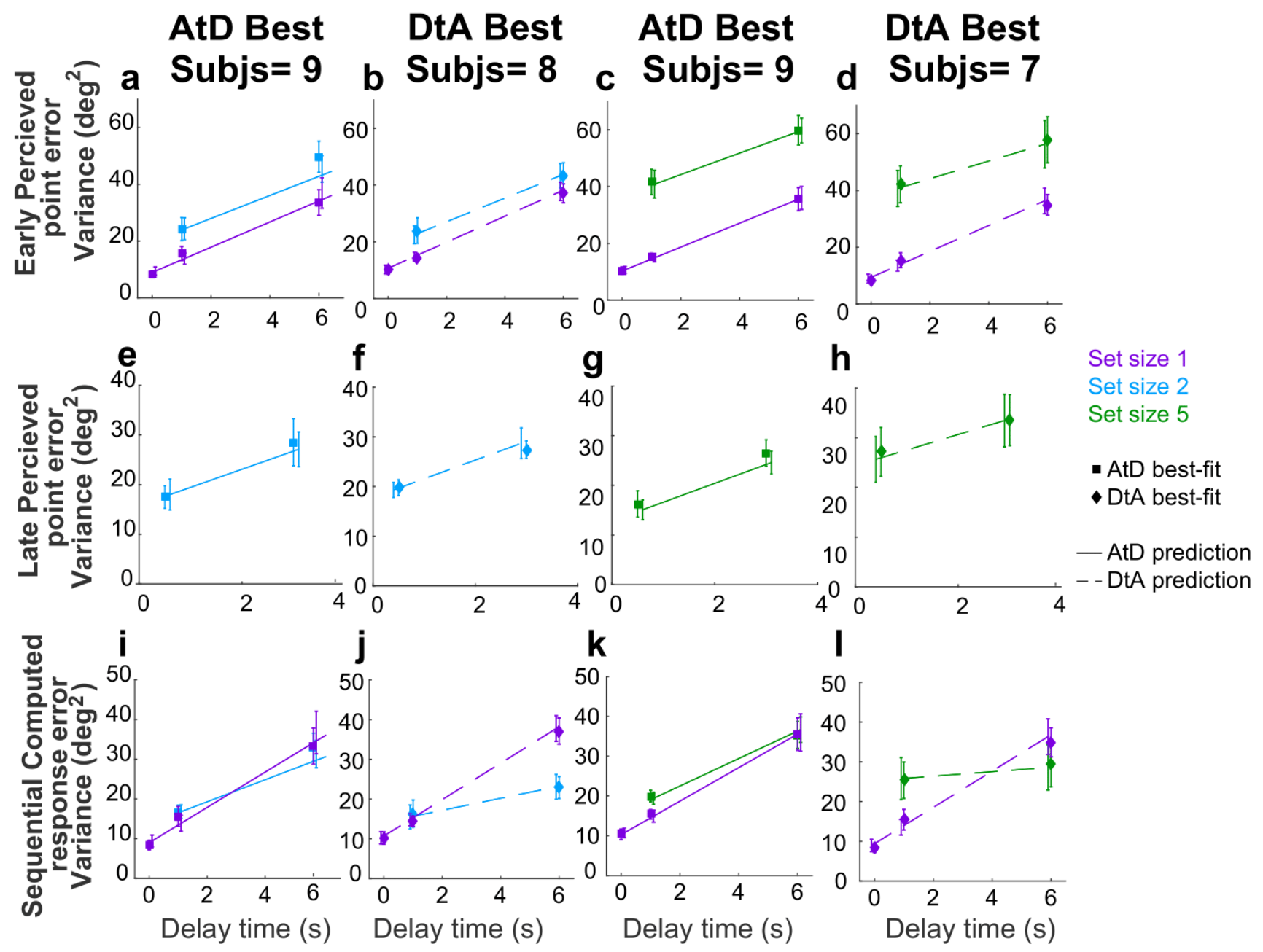

Figure 9. Comparison of model fits for the Sequential condition. Each panel shows the empirical variance of participant errors (points and error bars are mean \pm SEM data across participants) and model predictions (lines, using mean predicted variance from each participant's best-fitting parameters for the given model) for the participants best fit by the given model (AtD or DtA) for the given condition, as labeled above each Column. Panels a-d) errors for Ealy points in Perceived Sequential, e-h) errors for Late points in Sequential Perceived blocks. i-l) depict errors for Sequential Computed blocks. 


\section{Sequential condition strategy comparisons}

244 Across the population, participants had roughly equal tendencies to use either one the two

245 strategies (AtD or DtA) for the two set-size conditions (Fig. 10). Specifically, about an equal number of

246 participants were best fit by the AtD $(n=9)$ or DtA $(n=8)$ model for a set size of 2 (Wilcoxon signed-rank

247 two-sided test for the median difference in the log-likelihoods of fits of the two models to data from each

248 participant $=0, p=0.868)$. An approximately equal number of participants were also best fit and well fit by

249 the AtD $(n=9)$ or DtA $(n=7)$ model for a set size of 5 and neither model was more likely to be the better

250 fit across participants $(p=0.234)$. Participants not poorly fit at either set size were not significantly more

251 likely to be fit by either model across set sizes (Wilcoxon signed-rank two-sided test for identical median

252 log-likelihoods difference of fits of the two models across set size, $p=0.283$ ). Additionally, the log

253 likelihood difference of each model producing the data did not correlat e with age of participants (Fig.

$254 \mathrm{~S} 8 \mathrm{~b}$; Pearson correlation, $p>0.20$ ). In general, all of the participants lost fidelity in their representation of

255 a Computed value when it needed to consider sequentially presented information, as in many processes

256 of evidence accumulation. However, the dynamics of this degradation differed for the two strategies,

257 neither of which was more likely than the other for a given participant.

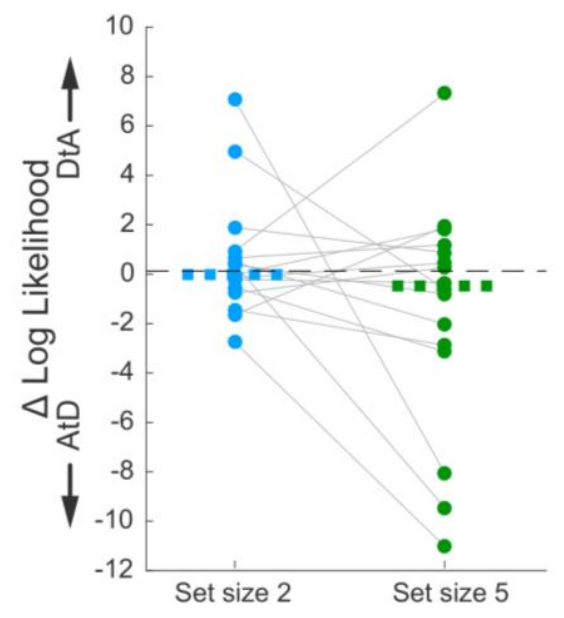

Figure 10. Difference in log likelihood per well-fit participant AtD and DtA fits. Negative values favor AtD. Each point represents the difference in fit log likelihoods for one participant and data from the same participant are connected across set sizes; horizontal bars are medians. Positive values favor DtA while negative values favor AtD. We failed to reject the null hypothesis (two-sided Wilcoxon signed rank test for $H_{0}$ : median $=0, p>0.05$ ) for both set sizes. 
$\underline{\text { Strategy comparisons across conditions }}$

The use of different strategies (i.e., those captured by the AtD and DtA models) did not appear to reflect a tendency of individual participants to use a particular strategy across different conditions.

261 Specifically, we used Fisher's exact test of independence based on set size across temporal conditions as

262 well as based on temporal conditions across set sizes to test whether individual participants were best fit

263 by the same model under different task conditions. We failed to reject the null hypothesis that there is no

264 relationship between a participant's strategy use across set size for both Simultaneous and Sequential

265 conditions (i.e., strategy use in set size 2 Simultaneous was not predictive of use in set size 5

266 Simultaneous, nor was it for Sequential conditions; $p=0.31$ and $p=1$ respectively). We also failed to reject

267 the null hypothesis that there is no relationship between a participant's strategy use across temporal

268 conditions for both set sizes 2 and 5 ( $p=0.54$ and $p=1$ respectively). Thus, we found that only under set

269 size 5 were Simultaneous conditions participants more likely to use one strategy (AtD) over the other

270 (DtA). In all other tested cases, participants were equally likely to use either strategy, and strategy use

271 was not predictive across conditions for individual participants.

\section{Discussion}

273

The goal of this study was to better understand if and how capacity and temporal limitations of

274 working memory affect human decision-making. We used a task that required participants to report

275 remembered spatial locations based on different numbers of objects and for different delay durations,

276 both of which are known to systematically affect the precision of memory reports (Bastos et al., 2018;

277 Cowan et al., 2008; Funahashi et al., 1989; Oberauer et al., 2016; Panichello et al., 2019; Ploner et al.,

278 1998; Schneegans \& Bays, 2018; White et al., 1994). We used two pairs of conditions to investigate these

279 effects across decision-making circumstances. The first condition was Perceptual versus Computed,

280 which allowed us to recapitulate previous findings of the effects of capacity and temporal limitations of

281 working memory for directly observed (perceptual) quantities and then extend those findings to the kind 
of computed quantity that is used as a decision variable for tasks that require integration or averaging to

283 reduce uncertainty (Brody \& Hanks, 2016; Gold \& Shadlen, 2007; Ratcliff et al., 2016; Shadlen \&

284 Shohamy, 2016; Summerfield \& Tsetsos, 2012). The second was Simultaneous versus Sequential

285 conditions, which extended our investigation to include the effects of working-memory limitations on

286 decision making under relatively simple conditions (i.e., when all relevant evidence was presented at

287 once) to the effects in a basic case of evidence accumulation over time (i.e., in which a new piece of

288 evidence is used to update a computed quantity).

Our primary finding was that computed variables based on either simultaneously or sequentially

290

presented information were susceptible to the same kinds of working-memory constraints as perceived

variables. These working-memory limitations corresponded to a decrease in precision over time, which

292

places critical constraints on the kinds of decision variables that are required to persist over time, such as

when decisions are delayed. This result appears to contradict previous findings that found no effect of

extra delays on the effectiveness of evidence accumulation for certain decisions (Liu et al., 2015;

295 Waskom \& Kiani, 2018). However, those studies used tasks with binary choices that required decision variables with less clear sensitivity to the kinds of working-memory effects we found in the context of a continuous, spatially based decision variable. Additionally, we found that increasing the number of decision-relevant points also decreased the accuracy of the continuous decision variable, although the nature of this effect was variable. More work is needed to fully characterize the conditions under which temporal and capacity limitations on the precision of working-memory representations affect decisions based on those representations. 
and then stores the Computed value. Its key prediction is that a Computed value should be susceptible to the same effects of working-memory limitations as a single remembered Perceptual value in simultaneous conditions. The second strategy, captured by the Diffuse-then-Average (DtA) model, stipulated that all individual values are stored in working memory until the time of decision. Its key prediction is that the overall rate of variance increase is inversely related to the number of items. We found that participants

311 tended to use an AtD strategy for the Simultaneous conditions with a relatively high load (five items),

312 but otherwise were roughly equally likely to use either strategy, including for all Sequential conditions.

314 found that for the Simultaneous condition, several individuals switched from using DtA for the smaller

315 set size to AtD for the larger set size, but we do not know if this switch was a consequence of their 316 personal working-memory capacities. From an optimality standpoint, DtA better preserves a computed

317 value compared to AtD for a given level of non-time-dependent noise and cost per storage item $(A)$, but 318 only if $A$ remains low (<1). It would be interesting to see if for more intermediate set sizes (i.e., 3 or 4 319 items) there is a reliable increase in the probability of a participant using AtD with a progression that 320 relates to other measures of the individual's working-memory capacity. Such future studies would more 321 definitively support the conclusion that increased working-memory load corresponds to an increased 322 tendency to discard information about individual samples and hold only the computed decision variable 323 in memory. Future studies should also examine other factors that might govern which strategy is used for 324 a given set of conditions. For example, participants in our study were instructed to report the average but 325 given no additional details about how to do so, nor given strong incentives for choosing any particular 326 strategy versus another. Future studies could provide more detailed instructions, incentives, and/or

327 feedback to better understand the flexibility with which these different strategies can be employed. 
over time. First, our DtA model assumed no interference between multiple items stored in memory. This

331 assumption is undoubtedly an oversimplification, given that storage of multiple points has been both

332 hypothesized and shown to create attraction and repulsion (Almeida et al., 2015; Kilpatrick, 2018; Wei

333 et al., 2012). Such directional drift can create a decrease in variance over time that could affect decision

334 variables that involve multiple quantities stored at once. Second, our DtA model also assumed that each

335 item was stored individually. Alternatively, items could have been discarded or merged (chunked)

336 (Kilpatrick, 2018; Wei et al., 2012), leading to different memory loads which could also affect

337 performance. Third, we did not find strong evidence that participant behavior could not be described by

338 the AtD or DtA model, but there was some evidence that our poorly fit subjects might have been using a

339 strategy that started out storing multiple points as in DtA but that combined the evidence into a single

340 variable midway through the delay in an AtD-like fashion. This kind of strategy would suggest extensive

341 flexibility in when and how evidence is incorporated into computed decision variables, thereby placing

342 potentially complex demands on working memory.

343 Both of our models were based on assumptions of a drifting memory representation. This random

344 drift is traditionally associated with attractor models of working memory (Bays, 2014; Compte, 2000;

345 Macoveanu et al., 2007; Wei et al., 2012) that have been used extensively to describe the underlying

346 neural mechanisms (Funahashi et al., 1989; Shafi et al., 2007; Takeda \& Funahashi, 2002; Wimmer et

347 al., 2014). In these models, neural network activity is induced by an external stimulus and then maintained

348 via excitatory connections of similarly tuned neurons and long-ranged inhibition. Random noise causes

349 the center of this activity (which represents the stimulus) to drift in a manner that, dependent on the

350 implementation, can depend on the delay duration, set size, and/or their interaction (Almeida et al., 2015;

351 Bays, 2014; Koyluoglu et al., 2017). A recent implementation even can naturally compute a running

352 average based on sequentially presented information (Esnaola-Acebes et al., 2021). Our results imply

353 that such models should be extended to support the flexible use of different strategies that govern when 
354 and how incoming information is used to form such averages. It will be interesting to see if such a flexible

355 model can account for neural activity in the dorsolateral prefrontal cortex (dlPFC), which includes

356 neurons with persistent activity that has been associated with both spatial working memory ${ }^{11,22-25}$ and the

357 formation of decisions based on an accumulation of evidence (Curtis \& D’Esposito, 2003; H. R. Heekeren

358 et al., 2006; Hauke R. Heekeren et al., 2008; Kim \& Shadlen, 1999; Lin et al., 2020; Philiastides et al.,

359 2011).

360 In conclusion, we found that in this spatial, continuous task, participant accuracy for both perceived

361 and computed values was subject to working-memory limitations of both time and capacity. Additionally, we

362 found behavior that was consistent with both the storage strategies we investigated. The fact that different

363 participants employed different strategies for storing a computed value (such as a decision variable) and that

364 these strategies have different consequences on overall accuracy has important implications for not only future

365 neural network models of working memory, but also for future computational models of decision-making. 


\section{Materials and Methods}

367 Human psychophysics behavioral task.

We tested 17 participants ( 4 male, 12 female, 1 chose not to answer; age range $=22-87 \mathrm{yrs}$ ). The task was created with PsychoPy3(Peirce et al., 2019) and distributed to participants via Pavlovia.com, which allowed participants to perform the task on their home computers after providing informed consent.

371 These protocols were reviewed by the University of Pennsylvania Institutional Review Board (IRB) and

372 determined to meet eligibility criteria for IRB review exemption authorized by 45 CFR 46.104, category 3732. Participants were instructed to sit one arm-length away from their computer screens during the experiment and to use the mouse to indicate choices. Each participant completed 1-2 sets of 4 blocks of trials in their own time. white fixation cross (1\% of the screen height). The participant was instructed to maintain fixation on this cross when not actively responding. The participant began each trial by placing the mouse over the cross and clicking, to allow for self-pacing and pseudo-fixation. Initiating a trial caused a white annulus of radius $25 \%$ of the screen height to appear. A block-specific memory array appeared $250 \mathrm{~ms}$ later, centered at an angle chosen uniformly and at random on the anulus. The array consisted of 1, 2, or 5 colored disks sized $1.5 \%$ screen in diameter. The angular difference between any two adjacent disks was at least $6^{\circ}$, and between the two most distal disks was at most $60^{\circ}$. The disks from clockwise to counter-clockwise were always presented in the same order: green, red, blue, magenta, and yellow. When fewer than five disks were presented, the latter colors were omitted. The consistent color ordering was intended to reduce errors caused by mis-binding of location and color. The angular differences between disks in an array was randomly selected from 5 preselected sets. The memory array remained on the screen for $0.5 \mathrm{~s}$, while 
fixation cross was replaced with a response cue that either matched a color of a disk in the memory array,

391 indicating a response to the remembered location of that disk, or was white, indicating a response to the

392 mean angle of all disks in the present trial. The response type varied by block (see below). The participant

393 then moved the mouse and clicked on the annulus at a position at which they remembered the requested

394 response. Feedback was then given indicating the correct location, the participant's response, and the

395 difference between the two.

We used four block-wise conditions: 1) Simultaneous Perceived blocks used arrays of 1, 2, or 5

disks presented simultaneously at the beginning of the trial. Participants were told in advance that they

398 would always be asked to report the location of one of the array disks but were not informed which one until the response period. The probed disk was picked randomly on each trial. 2) Simultaneous Computed blocks used arrays of 2 or 5 disks presented simultaneously at the beginning of the trial. Participants were

401 told in advance they would need to report the average angle of all disks shown in the present trial. 3)

402 Sequential Perceived blocks were identical to Simultaneous Perceived blocks, except only arrays of 2 or

4035 disks were used, and all but one of the disks (the counter-clockwise most) was presented at the beginning

404 of the trial. The final disk was presented for $0.5 \mathrm{~s}$ ending midway through the delay of 1 or $6 \mathrm{~s}$. Participants

405 were told in advance that the final disk would be presented in the middle of the delay for these blocks. 4)

406 Sequential Computed blocks were identical to Simultaneous Computed blocks, but with delayed 407 presentation of the final disk as in Sequential Perceived blocks. Again, participants were told in advance 408 that the final disk would be presented in the middle of the delay.

410 block contained 50 trials at each set size and each delay time, the order of which was randomized. 


\section{Basic analyses.}

Trials were excluded from analysis if the response was $>30^{\circ}$ from the correct angle. This cutoff

413 was based on assessment of the error distributions (Fig. S1-2); using a cutoff of $25^{\circ}$ did not noticeably

414 change the results. On average, $<10 \%$ of trials were excluded per delay condition per set size per block

415 (see Fig. S1-2). These trials were excluded to focus analysis on trials that were directed towards the

416 correct location and avoid lapses of attention and extreme motor errors. We investigated both the bias

417 and variance in participant responses, as follows.

418 We quantified bias as the mean error between the response and the true probed angle for each

419 participant and condition (positive/negative values imply errors that were systematically

420 counterclockwise/clockwise respectively). A Bonferroni-corrected two-sided $t$-test was used to assess

421 whether this mean response error was significantly different from zero across participants for each set

422 size, delay, response type and temporal presentation. Additionally, the mean error and confidence interval

423 for each subject were calculated for each condition (Fig. S3-6).

424 We quantified the variance of the error between the response and the true probed angle for each

425 participant and condition. We chose variance as opposed to other measures of dispersion for consistency

426 with our particle models (see below) in which variance scales linearly with delay. We examined effects

427 of set size, delay duration, and task context on response variability using a two-way repeated measures

428 ANOVA. On Simultaneous Perceived and Computed blocks, we used a 3 (delay duration: 0, 1, or 6 s) x

4293 (set size: 1, 2, or 5 disks) within-participant design. On Sequential Perceived blocks, we used a 2 (delay

430 duration: 1 or 6 s) x 3 (set size: 1, 2, or 5 disks) within-participants design for stimuli presented at the

431 beginning of the trial (Early) and a 2 (delay: 0.5 or 3 s) x 2 (set size: 2 or 5 disks) design for stimuli

432 presented halfway through the trial (Late). On Sequential Computed blocks, we used a 2 (delay duration:

4331 or 6 s) x 3 (set size: 1, 2, or 5 disks) within-participants design. When the comparison included set

434 size $=1$, data were always taken from the Simultaneous Perceived block. 
Model-based analyses.

Our models were based on principles of working memory that are well described by bump-

437 attractor network models (Compte, 2000; Laing \& Chow, 2001; Wimmer et al., 2014). In such models,

438 stimulus location is represented by a "bump" in activity from neurons tuned to that and similar locations.

439 These neurons recurrently activate each other, maintaining a bump of activity even after stimulus

440 cessation. However, because of the stochastic nature of neural activity and synaptic transmission(Faisal

441 et al., 2008), there is variability in which neurons have the most activity at any given time (and thus are

442 the center of the bump representing the stimulus). This variability in bump center corresponds to

443 variability in the location representation and a degradation of the memory representation over time. The

444 dynamics of this bump can be described as a diffusion process that obeys Brownian motion (Compte,

445 2000; Kilpatrick, 2018; Kilpatrick et al., 2013; Laing \& Chow, 2001). We used this simplified description

446 in our models as follows.

447 Perceived values in working memory.

448 A single point (i.e., the central spatial location of a single disk), $x_{1}$, is assumed to be represented

449 in working memory by $\hat{x}_{t, 1}$, where $t$ represents the time since the removal of the stimulus. We assume that

$450 \hat{x}_{t, 1}$ evolves like a sample from a Brownian-motion process. Specifically, when $x_{1}$ is observed, it is

451 encoded with some perceptual noise, $\eta^{p}$. Therefore, at time zero, $\hat{x}_{0,1} \sim N\left(x\right.$, $\left.\eta^{p}\right)$. This representation

452 accumulates noise over time with some diffusion constant, $\sigma_{l}^{2}$, further degrading the representation of

$453 \hat{x}_{t, 1}$ from $x_{1}$ such that $\hat{x}_{t, 1} \sim N\left(x_{1}, \eta^{p}+t^{*} \sigma_{1}^{2}\right)$. There is additional motor noise in the participant's report, $r_{t, 1}$,

454 and we denote the variance of this motor noise by $\eta^{m}$. Mathematically, it is equivalent to add the motor

455 noise at the beginning or the end of the diffusion of $\hat{x}_{t, 1}$ when considering the report, $r_{t, 1}$. In our model,

456 we thus represent the sum of the perceptual and motor noise as a single, non-time-dependent noise term.

457 Hence, we show simulated trajectories of $\hat{x}_{t, 1}$ in Fig. 2 a with an initial variance of $\eta_{1}=\eta^{p}+\eta^{m}$ so that at 458 time $t$ the report, $r_{t, 1}$ is the current angle of the trajectory. Therefore: 


$$
r_{t, 1} \sim N\left(x_{1}, \eta_{1}+t^{*} \sigma_{1}^{2}\right)
$$

461 et al., 2009; Brady \& Alvarez, 2015; Koyluoglu et al., 2017; Wei et al., 2012). We therefore assume that

462 the sum of the initial perceptual noise and final motor noise, with variance denoted by $\eta_{N}$, can depend on

463 the number of disks, $N$. Moreover we describe, $\hat{x}_{t, n}$, the representation of the $n^{\text {th }}$ item at time $t$, by a

464 normal distribution with a diffusion constant that is potentially higher than for a single point. We assume

465 that this new diffusion constant $\sigma_{\mathrm{N}}^{2}$, equals $\sigma_{1}^{2} * N^{A}$ and thus scales as a power of the total number of

466 stimuli, $N$, held in memory (Bays et al., 2009; Bays \& Husain, 2008; Wei et al., 2012), and is proportional

467 to the diffusion constant corresponding to a single stimulus representation, $\sigma_{1}^{2}$. Therefore:

$$
r_{t, n} \sim N\left(x_{n}, \eta_{N}+t^{*} \sigma_{l}^{2 *} N^{A}\right)
$$

All representations in a set of size $N$ share the same magnitude of non-time-dependent noise, $\eta_{N}$, between the true and reported location (the error, $\left.e_{t, n}\right)$ is

$$
\begin{aligned}
& e_{t, 1} \sim N\left(0, \eta_{1}+t * \sigma_{1}^{2}\right) \\
& e_{t, n} \sim N\left(0, \eta_{N}+t * \sigma_{1}^{2} * N^{A}\right)
\end{aligned}
$$
memoranda is illustrated in Fig. 2 b. 
assume that the representation of the average has the same non-time-dependent noise as a single point, because there could be additional noise from inaccurately averaging multiple points or conversely a reduction in overall noise resulting from the averaging of multiple random variables (constituent points).

485 We denote the variance of the non-time-dependent noise for the Computed mean by $\eta_{M N}$. The difference 486 between the true mean of $N$ stimuli and the mean reported at time $t$ is therefore,

$$
e_{t, m N A t D} \sim N\left(0, \eta_{M N A t D}+t^{*} \sigma_{1}^{2}\right)
$$

Diffuse-then-Average (DtA) Simultaneous model.

For this model, the individual perceived points are stored as individual, independently diffusing particles and then averaged at the end of the trial. Thus, the diffusion constant of the Computed value is

491 the variance of the average of $N$ random variables each with the diffusion constant $\sigma_{1}^{2 *} N^{A}$, resulting in

492 an effective diffusion constant for the Computed value of $\sigma_{M N}{ }^{2}=\sigma_{1}^{2 *} N^{A} / N$, where the division by $N$ arises

493 from averaging. Again, we allow for a free non-time-dependent-noise term because of the uncertain

494 effects of the averaging calculation itself. For this model, the error in the reported location at time $t$ of 495 the average of the mean, $M$, of $N$ points, $e_{t, M N}$, is:

$$
e_{t, m N D t A} \sim N\left(0, \eta_{M N D t A}+t^{*} \sigma_{1}^{2} * N^{A} / N\right)
$$
strategy results in a lower diffusion constant for a Computed value than predicted by the AtD model and results in a smaller average reporting error (see Fig. 2c). If $A>1$, then AtD results in the lower diffusion

501 constant and thus a lower average reporting error. However, we did not find that participants necessarily 


\section{Sequentially presented values in working memory.} was presented halfway through the delay (Late point). Therefore, both our modeling assumes that the diffusion constant for the representation of the $N-1$ early stimuli change with the addition of the $N^{\text {th }}$ point.

507 In contrast, the representation of the Late stimulus diffuses only for half of the delay time, $T$, as shown 508 in Fig. 2c. We formalize this process by the following model for the report error of the Early $\left(e_{T, N E}\right)$, and 509 Late $\left(e_{T, N L}\right)$ stimuli:

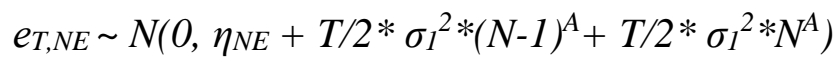

$$
\begin{aligned}
& e_{T, N L} \sim N\left(0, \eta_{N L}+T / 2 * \sigma_{l}^{2} * N^{A}\right)
\end{aligned}
$$

513 Early and Late points, $\eta_{N E}$ and $\eta_{N L}$, respectively.

$\underline{\text { AtD Sequential model: }}$

This model assumes that the Early points are averaged immediately and stored as a single point.

516 At $t=T / 2$, the Late point is presented and the stimulus is immediately combined, through appropriate

517 weighted averaging, with the mean of the Early points. This new mean again diffuses with the same 518 accumulating noise as a single point, as depicted in Fig. 2d. Therefore:

$$
e_{T, M N-s e q A t D} \sim N\left(0, \eta_{M N-S e q A t D}+((N-1) / N)^{2} * T / 2 * \sigma_{1}^{2}+T / 2 * \sigma_{l}^{2}\right)
$$

521 has non-time-dependent noise, which is absorbed in the $\eta_{M N-S e q}$ term. The first time-dependent term, $((N-$

$5221) / N)^{2} * T / 2 * \sigma_{l}^{2}$, results from the appropriate weighted averaging of the mean of the Early points (time-

523 dependent noise of $T / 2 * \sigma_{1}^{2}$ ) with the Late point (time-dependent noise=0). The final term, $T / 2 * \sigma_{1}^{2}$, is 524 the diffusion of the resultant mean until the end of the delay. 
DtA Sequential model:

This model assumes that the representations of all $N$ points diffuse as they are presented, resulting

527 in $N-1$ points described by eq. $6 \mathrm{a}$ and one point described by eq. $6 \mathrm{~b}$. These points are then averaged at

528 the end of the delay, resulting in an overall error of:

$$
e_{T, M N-s e q D t A} \sim N\left(0, \eta_{M N-S e q D t A}+\left(T / 2 * \sigma_{1}^{2} * N^{A}+\ldots\right.\right.
$$

$$
\left.\left.(N-1)\left[T / 2 * \sigma_{1}^{2} *(N-1)^{A}+T / 2 * \sigma_{1}^{2} * N^{A}\right]\right) / N^{2}\right)
$$

531 where the constant noise terms from the Early and Late points are absorbed in the $\eta_{M N-S e q D t A}$ term, the

532 next term $T / 2 * \sigma_{1}{ }^{2} * N$ is the diffusion in the representation of the last disk shown, and the remaining terms

533 arise from the first $N-1$ disks shown. The effect of this averaging on the effective diffusion constant are

534 shown in Fig. 2d.

535 Model fitting.

To fit both the AtD and DtA models to data from the Perceived and Computed blocks we had to estimate 5 parameters: 1$)$ the non-time-dependent noise of a single point $\left.\left(\eta_{1}\right), 2\right)$ the diffusion noise of a

538 single point $\left.\left(\sigma_{l}^{2}\right), 3\right)$ the non-time-dependent noise of $N$ points $\left.\left(\eta_{N}\right), 4\right)$ the exponent of storing $N$ points

539 (A), and 5) the non-time-dependent noise of the mean $\left(\eta_{M N(A t D \text { or } D t A)}\right)$. We fit these models for $N=2$ and $540 N=5$ separately using trials from the following conditions: Perceived Simultaneous delays of 1, 3, and 6 541 s, with array sizes 1 (eq. 3a) and $N$ (eq. 3b); Computed Simultaneous, delays of 1, 3, and 6 s, with array 542 size $N$ (eq. 4 for AtD, eq. 5 for DtA).

543 Data from the Sequential Perceived and Sequential Computed blocks were fit to the AtD and DtA

544 models with 6 parameters. The additional parameter accounted for differences in the non-time-dependent 545 noise for Early and Late points. We fit these models for $N=2$ and $N=5$ separately using trials from the 546 following conditions: Perceived, delay 1, 3, and 6 s with array sizes 1 (eq. 3a); Sequential Perceived, 
547 delay of 3 and 6 s, array size $N$ for both Early (eq. 6a) and Late (eq. 6b) points; Sequential Computed,

548 delay of 3 and 6 s, array size $N$ (eq. 7 for AtD or eq. 8 for DtA).

Because the mean error for each individual participant was not always zero, when fitting the AtD

550

and DtA models we used the empirical mean error from the condition being fitted as a fixed bias term in

551 the model. Mean error and confidence intervals for each participant for each condition are shown in Fig.

552 S3-6.

We obtained separate maximum-likelihood fits for AtD and DtA models for each individual

554 participant, using the function fmincon in MATLAB to minimize the summed negative log likelihood of

555 obtaining the observed errors for a given condition according to the above equations. Initial parameter

556 values were randomized and the fitting repeated to avoid local minima. Because all models within a given

557 condition had the same number of parameters, we compared log likelihoods to determine the best-fitting

558 model for a given participant. Because the number of parameters are the same, comparing likelihoods

559 produces equivalent model selection to BIC or AIC.

560 Goodness-of-fit.

561 To assess how well each participant's data matched the assumptions of the AtD and DtA models,

562 we also fit a line to the variances of response errors across delays for a given condition for a given

563 participant to obtain empirical estimates of the various diffusion constants (e.g., slope of lines in Fig. 2b;

564 empirical estimate of a Perceived value, $\hat{\sigma}_{N}^{2}$, for set size $N$; empirical estimate of a Computed value,

$565 \hat{\sigma}_{M N^{2}}{ }^{2}$, set size $N$ ). These empirical estimates of the diffusion constants did not enforce the relationships

566 imposed by the AtD and DtA models between the different diffusion constants in each model

567 respectively. We compared the relationships of these empirical estimates of diffusion constants to the

568 relationships assumed by our models. 
AtD Simultaneous expected diffusion constant relationship:

571 same diffusion constant as a single value. Thus:

$$
\sigma_{1}^{2}-\sigma_{M N}^{2}=0
$$

573 DtA Simultaneous expected diffusion constant relationship:

points each diffusing with a constant of $\sigma_{N}^{2}$. Thus:

$$
\sigma_{N}^{2} / N-\sigma_{M N}^{2}=0
$$

AtD Sequential expected diffusion constant relationship:

$$
\left[(N-1)^{2}+N^{2}\right] /\left(2 N^{2}\right) * \sigma_{1}^{2}-\sigma_{M N}^{2}=0
$$
increases as $\left.T / 2 * \sigma_{1}^{2} * N^{A}+(N-1)\left[T / 2 * \sigma_{1}^{2} *(N-1)^{A}+T / 2 * \sigma_{1}^{2} * N^{A}\right]\right) / N^{2}$ (eq. 8). By eq. 6a, the diffusion constant for an Early Perceived point, ${\sigma_{N E}}^{2}$, is $\left[0.5^{*} \sigma_{1}^{2} *(N-1)^{A}+0.5^{*}{\sigma_{1}}^{2 *} N^{A}\right]$ and by eq. $6 \mathrm{~b}$, the diffusion constant for a Late Perceived point, $\sigma_{N L}^{2}$, is $\sigma_{1}^{2} * N^{A}$. Factoring out $\mathrm{T}$ and substituting gives the diffusion constant for the Computed mean, $\sigma_{M N}^{2}=\left(0.5 \sigma_{N L}^{2}+(N-1) * \sigma_{N E}^{2}\right) / N^{2}$. Thus:

$$
\left(0.5^{*} \sigma_{N L}^{2}+(N-1) * \sigma_{N E}^{2}\right) / N^{2}-\sigma_{M N}^{2}=0
$$

To assess how well the relationships between participant empirical estimates of the diffusion 590 constants matched these assumptions, for each participant we simulated 1000 iterations of a participant 
591 performing the task using the best-fit model for the given true participant and the maximum likelihood

592 estimate parameters for that participant. We then estimated the empirical diffusion constants for each of

593 these iterations as we did for our participants, namely fitting a line to the measured variance of the

594 simulated errors across delays, for each condition and iteration. Our 1000 simulations gave us an expected

595 range around the expected diffusion constant relationships detailed in eq. 9-12 to compare to our

596 participants' empirical diffusion constant relationships. Participants whose empirical diffusion constant

597 relationships fell within the central $95 \%$ of the simulated expected range were considered well fit by their

598 model. 


\section{Acknowledgements}

599 We thank Adrian Radillo and Gaia Tavoni for their discussions early in the development of this project,

600 particularly regarding early formulations of the models used here and task structure.

\section{Competing Interests}

602 The authors declare no competing interests

\section{References}

Almeida, R., Barbosa, J., \& Compte, A. (2015). Neural circuit basis of visuo-spatial working memory precision: a computational and behavioral study. Journal of Neurophysiology, 114(3), 1806-1818. https://doi.org/10.1152/jn.00362.2015

Bastos, A. M., Loonis, R., Kornblith, S., Lundqvist, M., \& Miller, E. K. (2018). Laminar recordings in frontal cortex suggest distinct layers for maintenance and control of working memory. Proceedings of the National Academy of Sciences of the United States of America, 115(5), 1117-1122. https://doi.org/10.1073/pnas.1710323115

Bays, P. M. (2014). Noise in neural populations accounts for errors in working memory. The Journal of Neuroscience: The Official Journal of the Society for Neuroscience, 34(10), 3632-3645. https://doi.org/10.1523/JNEUROSCI.3204-13.2014

Bays, P. M., Catalao, R. F. G., \& Husain, M. (2009). The precision of visual working memory is set by allocation of a shared resource. Journal of Vision, 9(10), 7.1-11. https://doi.org/10.1167/9.10.7

Bays, P. M., \& Husain, M. (2008). Dynamic shifts of limited working memory resources in human vision. Science, 321(5890), 851-854. https://doi.org/10.1126/science.1158023

Bernacchia, A., Seo, H., Lee, D., \& Wang, X. J. (2011). A reservoir of time constants for memory traces in cortical neurons. Nature Neuroscience, 14(3), 366-372. https://doi.org/10.1038/nn.2752

Brady, T. F., \& Alvarez, G. A. (2015). Contextual effects in visual working memory reveal hierarchically structured memory representations. Journal of Vision, 15(15), 6. https://doi.org/10.1167/15.15.6

Brody, C. D., \& Hanks, T. D. (2016). Neural underpinnings of the evidence accumulator. In Current Opinion in Neurobiology (Vol. 37, pp. 149-157). Elsevier Ltd.

https://doi.org/10.1016/j.conb.2016.01.003

Compte, A. (2000). Synaptic Mechanisms and Network Dynamics Underlying Spatial Working Memory in a Cortical Network Model. Cerebral Cortex, 10(9), 910-923. https://doi.org/10.1093/cercor/10.9.910

Constantinidis, C., Funahashi, S., Lee, D., Murray, J. D., Qi, X.-L., Wang, M., \& Arnsten, A. F. T. 
(2018). Persistent Spiking Activity Underlies Working Memory. The Journal of Neuroscience : The Official Journal of the Society for Neuroscience, 38(32), 7020-7028.

https://doi.org/10.1523/JNEUROSCI.2486-17.2018

Cowan, N., Morey, C. C., Chen, Z., Gilchrist, A. L., \& Saults, J. S. (2008). Theory and Measurement of Working Memory Capacity Limits. In Psychology of Learning and Motivation - Advances in Research and Theory (Vol. 49, pp. 49-104). Academic Press. https://doi.org/10.1016/S00797421(08)00002-9

Curtis, C. E., \& D'Esposito, M. (2003). Persistent activity in the prefrontal cortex during working memory. In Trends in Cognitive Sciences (Vol. 7, Issue 9, pp. 415-423). Elsevier Ltd. https://doi.org/10.1016/S1364-6613(03)00197-9

Esnaola-Acebes, J. M., Roxin, A., \& Wimmer, K. (2021). Bump attractor dynamics underlying stimulus integration in perceptual estimation tasks. BioRxiv, 2021.03.15.434192.

https://doi.org/10.1101/2021.03.15.434192

Faisal, A. A., Selen, L. P. J., \& Wolpert, D. M. (2008). Noise in the nervous system. In Nature Reviews Neuroscience (Vol. 9, Issue 4, pp. 292-303). Nature Publishing Group. https://doi.org/10.1038/nrn2258

Funahashi, S., Bruce, C. J., \& Goldman-Rakic, P. S. (1989). Mnemonic Coding of Visual Space in the Monkey's Dorsolateral Prefrontal Cortex. In JOURNALOFNEUROPHYSIOLOGY (Vol. 6, Issue 2). www.physiology.org/journal/jn

Gold, J. I., \& Shadlen, M. N. (2007). The Neural Basis of Decision Making. Annual Review of Neuroscience, 30(1), 535-574. https://doi.org/10.1146/annurev.neuro.29.051605.113038

Gold, J. I., \& Stocker, A. A. (2017). Visual Decision-Making in an Uncertain and Dynamic World. In Annual Review of Vision Science (Vol. 3, pp. 227-250). Annual Reviews Inc. https://doi.org/10.1146/annurev-vision-111815-114511

Heekeren, H. R., Marrett, S., Ruff, D. A., Bandettini, P. A., \& Ungerleider, L. G. (2006). Involvement of human left dorsolateral prefrontal cortex in perceptual decision making is independent of response modality. Proceedings of the National Academy of Sciences of the United States of America, 103(26), 10023-10028. https://doi.org/10.1073/pnas.0603949103

Heekeren, Hauke R., Marrett, S., \& Ungerleider, L. G. (2008). The neural systems that mediate human perceptual decision making. In Nature Reviews Neuroscience (Vol. 9, Issue 6, pp. 467-479). Nature Publishing Group. https://doi.org/10.1038/nrn2374

Kilpatrick, Z. P. (2018). Synaptic mechanisms of interference in working memory. Scientific Reports, 8(1), 1-20. https://doi.org/10.1038/s41598-018-25958-9

Kilpatrick, Z. P., Ermentrout, B., \& Doiron, B. (2013). Optimizing working memory with heterogeneity of recurrent cortical excitation. The Journal of Neuroscience: The Official Journal of the Society for Neuroscience, 33(48), 18999-19011. https://doi.org/10.1523/JNEUROSCI.1641-13.2013

Kim, J. N., \& Shadlen, M. N. (1999). Neural correlates of a decision in the dorsolateral prefrontal cortex 
of the macaque. Nature Neuroscience, 2(2), 176-185. https://doi.org/10.1038/5739

Koyluoglu, O. O., Pertzov, Y., Manohar, S., Husain, M., \& Fiete, I. R. (2017). Fundamental bound on the persistence and capacity of short-term memory stored as graded persistent activity. ELife, 6 . https://doi.org/10.7554/eLife.22225

Laing, C. R., \& Chow, C. C. (2001). Stationary bumps in networks of spiking neurons. Neural Computation, 13(7), 1473-1494. https://doi.org/10.1162/089976601750264974

Lin, Z., Nie, C., Zhang, Y., Chen, Y., \& Yang, T. (2020). Evidence accumulation for value computation in the prefrontal cortex during decision making. Proceedings of the National Academy of Sciences of the United States of America, 117(48), 30728-30737. https://doi.org/10.1073/pnas.2019077117

Liu, A. S. K., Tsunada, J., Gold, J. I., \& Cohen, Y. E. (2015). Temporal integration of auditory information is invariant to temporal grouping cues. ENeuro, 2(2). https://doi.org/10.1523/ENEURO.0077-14.2015

Macoveanu, J., Klingberg, T., Tegnér, J., Macoveanu, J., Tegnér, · J, Klingberg, T., \& Tegnér, J. (2007). Neuronal firing rates account for distractor effects on mnemonic accuracy in a visuo-spatial working memory task. Biol Cybern, 96, 407-419. https://doi.org/10.1007/s00422-006-0139-8

Oberauer, K., Farrell, S., Jarrold, C., \& Lewandowsky, S. (2016). What limits working memory capacity? Psychological Bulletin, 142(7), 758-799. https://doi.org/10.1037/bul0000046

Panichello, M. F., DePasquale, B., Pillow, J. W., \& Buschman, T. J. (2019). Error-correcting dynamics in visual working memory. Nature Communications, 10(1), 3366. https://doi.org/10.1038/s41467019-11298-3

Peirce, J., Gray, J. R., Simpson, S., MacAskill, M., Höchenberger, R., Sogo, H., Kastman, E., \& Lindeløv, J. K. (2019). PsychoPy2: Experiments in behavior made easy. Behavior Research Methods, 51(1), 195-203. https://doi.org/10.3758/s13428-018-01193-y

Philiastides, M. G., Auksztulewicz, R., Heekeren, H. R., \& Blankenburg, F. (2011). Causal role of dorsolateral prefrontal cortex in human perceptual decision making. Current Biology, 21(11), 980983. https://doi.org/10.1016/j.cub.2011.04.034

Ploner, C. J., Gaymard, B., Rivaud, S., Agid, Y., \& Pierrot-Deseilligny, C. (1998). Temporal limits of spatial working memory in humans. European Journal of Neuroscience, 10(2), 794-797. https://doi.org/10.1046/j.1460-9568.1998.00101.x

Ratcliff, R., Smith, P. L., Brown, S. D., \& McKoon, G. (2016). Diffusion Decision Model: Current Issues and History. In Trends in Cognitive Sciences (Vol. 20, Issue 4, pp. 260-281). Elsevier Ltd. https://doi.org/10.1016/j.tics.2016.01.007

Riley, M. R., \& Constantinidis, C. (2015). Role of Prefrontal Persistent Activity in Working Memory. Frontiers in Systems Neuroscience, 9, 181. https://doi.org/10.3389/fnsys.2015.00181

Schneegans, S., \& Bays, P. M. (2018). Drift in Neural Population Activity Causes Working Memory to Deteriorate Over Time. The Journal of Neuroscience: The Official Journal of the Society for 
Neuroscience, 38(21), 4859-4869. https://doi.org/10.1523/JNEUROSCI.3440-17.2018

Shadlen, M. N. N., \& Shohamy, D. (2016). Decision Making and Sequential Sampling from Memory. In Neuron (Vol. 90, Issue 5, pp. 927-939). Cell Press. https://doi.org/10.1016/j.neuron.2016.04.036

Shafi, M., Zhou, Y., Quintana, J., Chow, C., Fuster, J., \& Bodner, M. (2007). Variability in neuronal activity in primate cortex during working memory tasks. Neuroscience, 146(3), 1082-1108. https://doi.org/10.1016/J.NEUROSCIENCE.2006.12.072

Summerfield, C., \& Tsetsos, K. (2012). Building bridges between perceptual and economic decisionmaking: Neural and computational mechanisms. In Frontiers in Neuroscience (Vol. 6, Issue MAY). Front Neurosci. https://doi.org/10.3389/fnins.2012.00070

Takeda, K., \& Funahashi, S. (2002). Prefrontal Task-Related Activity Representing Visual Cue Location or Saccade Direction in Spatial Working Memory Tasks. https://doi.org/10.1152/jn.00249.2001

Waskom, M. L., \& Kiani, R. (2018). Decision Making through Integration of Sensory Evidence at Prolonged Timescales. Current Biology, 1-7. https://doi.org/10.1016/j.cub.2018.10.021

Wei, Z., Wang, X.-J., \& Wang, D.-H. (2012). From Distributed Resources to Limited Slots in MultipleItem Working Memory: A Spiking Network Model with Normalization. Journal of Neuroscience, 32(33), 11228-11240. https://doi.org/10.1523/JNEUROSCI.0735-12.2012

White, J. M., Sparks, D. L., \& Stanford, T. R. (1994). Saccades to remembered target locations: an analysis of systematic and variable errors. Vision Research, 34(1), 79-92.

https://doi.org/10.1016/0042-6989(94)90259-3

Wimmer, K., Nykamp, D. Q., Constantinidis, C., \& Compte, A. (2014). Bump attractor dynamics in prefrontal cortex explains behavioral precision in spatial working memory. Nature Neuroscience, 17(3), 431-439. https://doi.org/10.1038/nn.3645 


\section{Supplemental Figures}
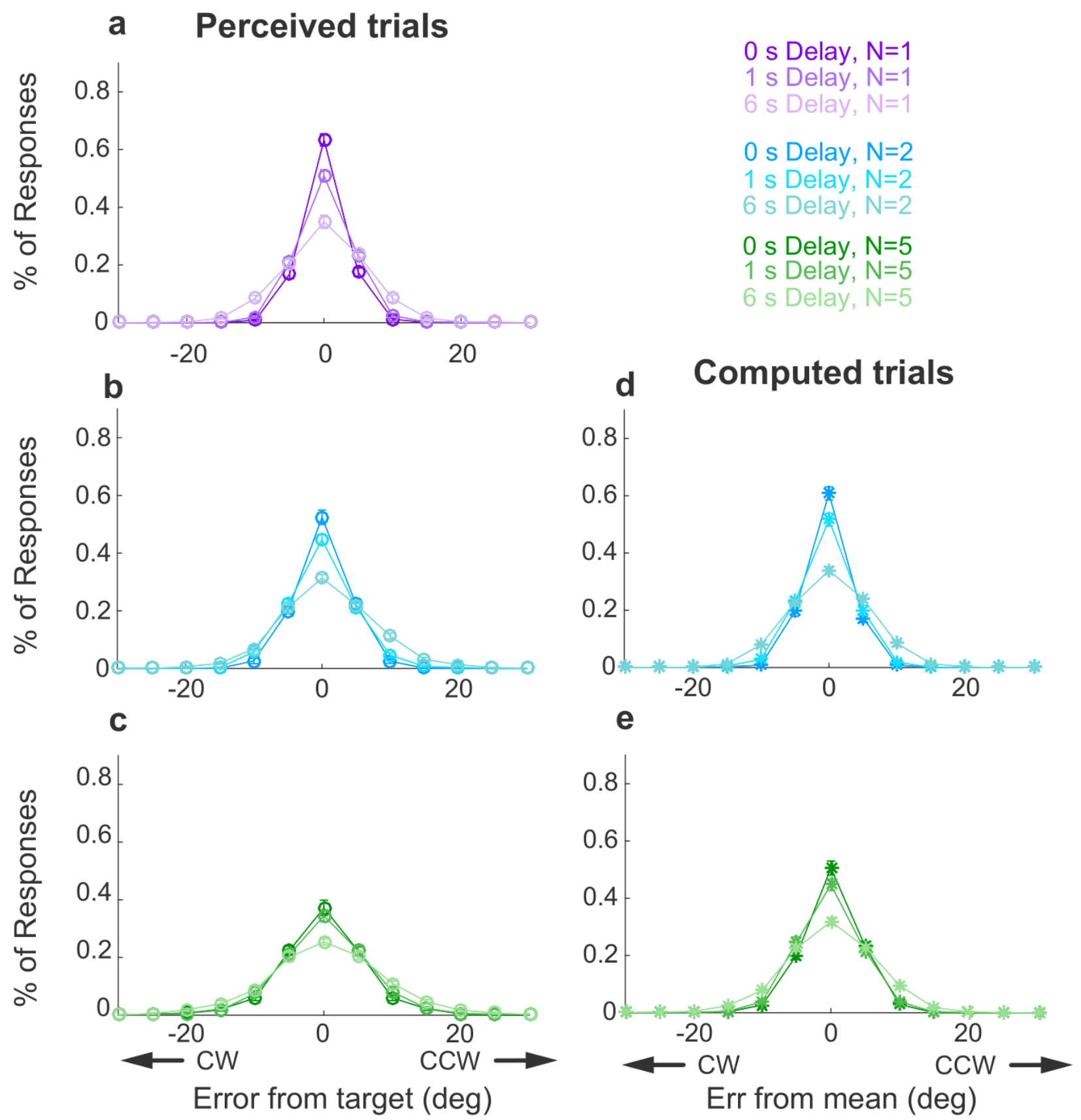

Supplemental Figure S1: Full error distributions in Simultaneous conditions. Each panel shows a histogram of mean error for different delays (colors, as indicated) for Perceived trials (left column: a) set $\operatorname{size}=1 ; \boldsymbol{b}$ ) $\operatorname{set} \operatorname{size}=2 ; \boldsymbol{c}$ ) set $\operatorname{size}=5$ ) and for Computed trials (right column; $\boldsymbol{d}$ ) set size $=2 ; \boldsymbol{e}$ ) set size $=5$ ). In each panel, points and error bars are mean \pm SEM across all participants. Note that in all cases, $95 \%$ of the distributions fall between $-30^{\circ}$ and $30^{\circ}$, justifying our exclusion of larger errors as offtarget responses. 

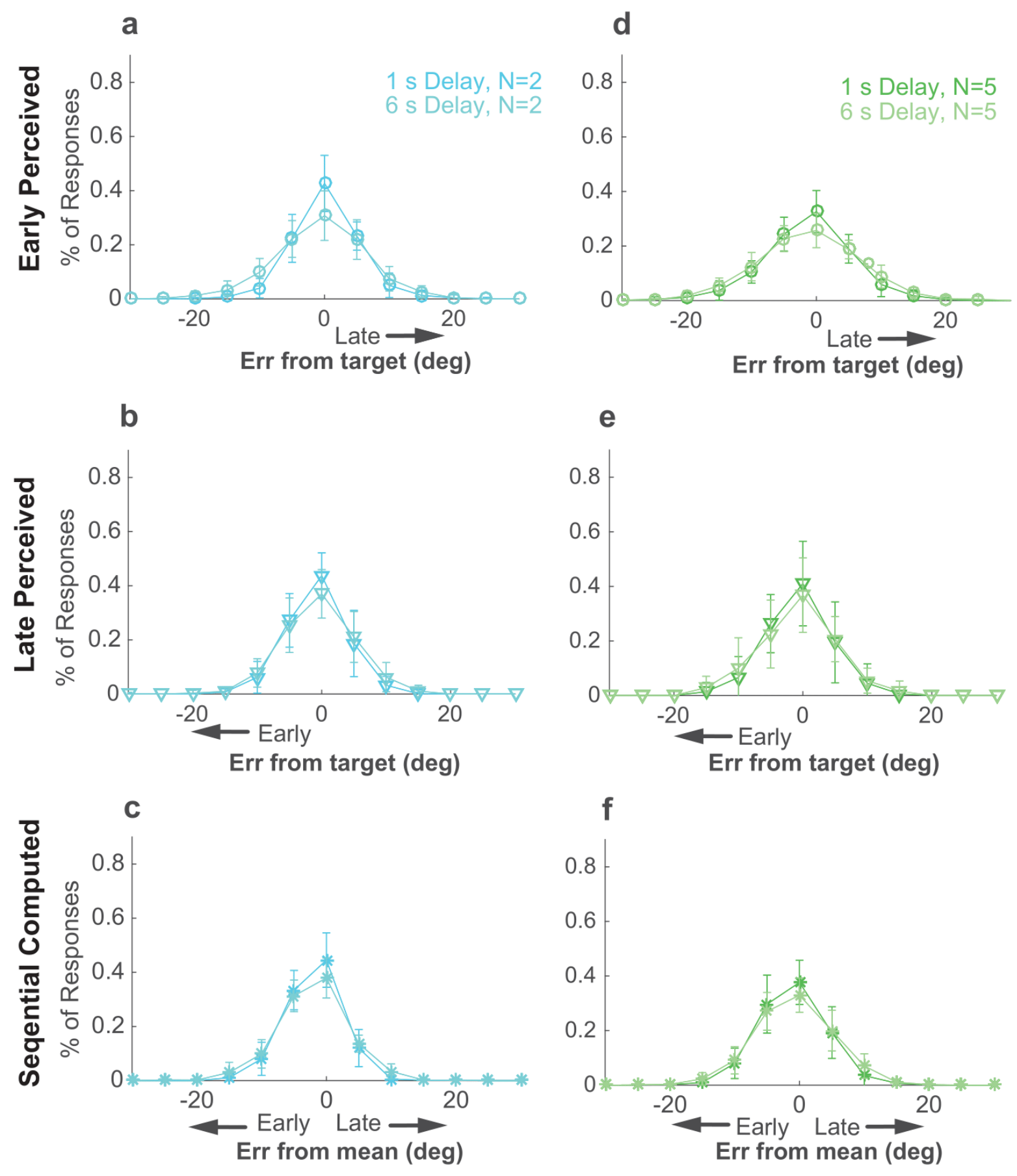

Supplemental Figure S2: Full error distributions in Sequential conditions. a) Histogram of mean Early Perceptual error for different delays for set size 2. b) Histogram of mean Late Perceptual error for different delays for set size 2. c) Histogram of mean Computed error for different delays for set size 2. d-f) As in a-c but for set size 5. In each panel, points and error bars are mean \pm SEM across participants. Note that in all cases, $95 \%$ of the distributions fall between $-30^{\circ}$ and $30^{\circ}$, justifying our exclusion of larger errors as off-target responses. 

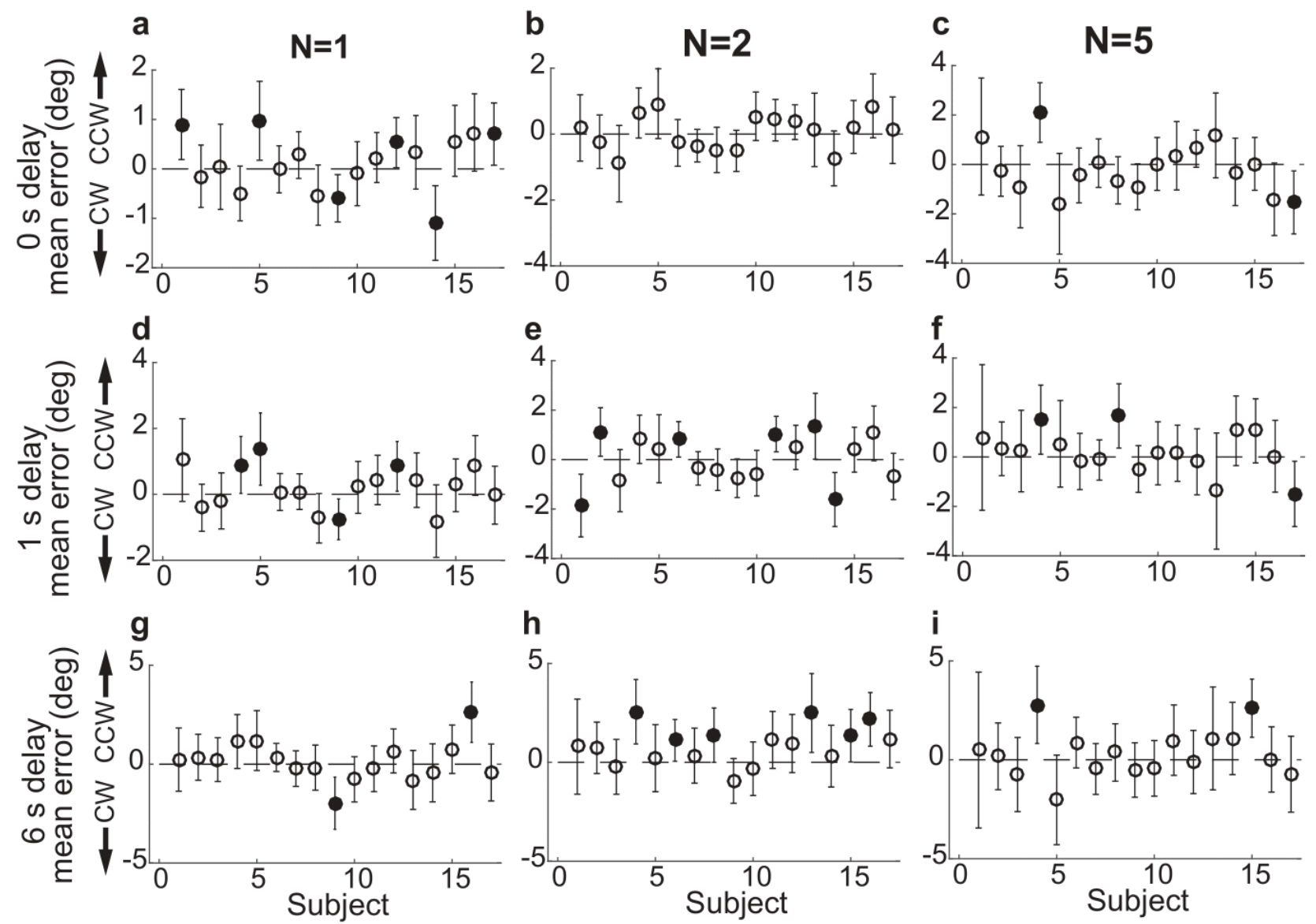

Supplemental Figure S3: Subject-wise mean response error in the Simultaneous Perceived condition. a) Delay $=0 \mathrm{~s}$, set $\operatorname{size}=1$. b) Delay $=0$, set size $=2$. c) Delay $=0 \mathrm{~s}$, set size $=5$. $\mathbf{d}-\mathbf{f}$ ) as in $\mathbf{a}-\mathbf{c}$ but for delay of $1 \mathrm{~s}$. $\mathbf{g}-\mathbf{i}$ ) as in $\mathbf{a}-\mathbf{c}$ but for delay of $6 \mathrm{~s}$. In all panels, errorbars are $\pm 95 \%$ confidence intervals. Filled points indicate that 0 is not included in the confidence interval; i.e., there is bias in subject errors. 

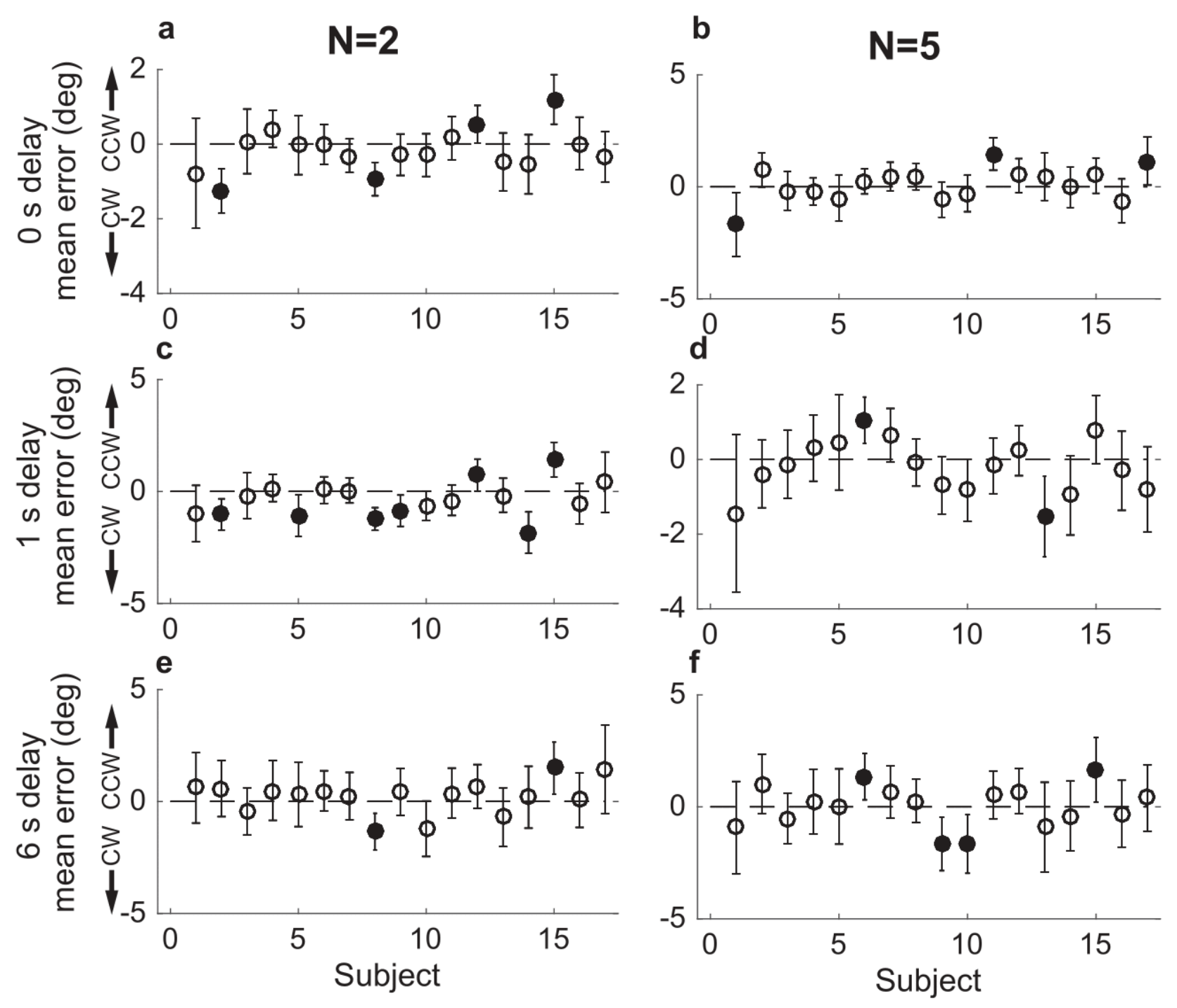

Supplemental Figure S4: Subject-wise mean error in the Simultaneous Computed condition. a) Delay $=0 \mathrm{~s}$, set size $=2$. b) Delay of 0 , set size $=5$. $\mathbf{c}-\mathbf{d}$ ) as in $\mathbf{a}-\mathbf{b}$ but for delay of $1 \mathrm{~s}$. e--f) as in $\mathbf{a}-\mathbf{b}$ but for delay of $6 \mathrm{~s}$. In all panels, errorbars are $\pm 95 \%$ confidence intervals. Filled points indicate that 0 is not included in the confidence interval; i.e., there is bias in subject errors. 

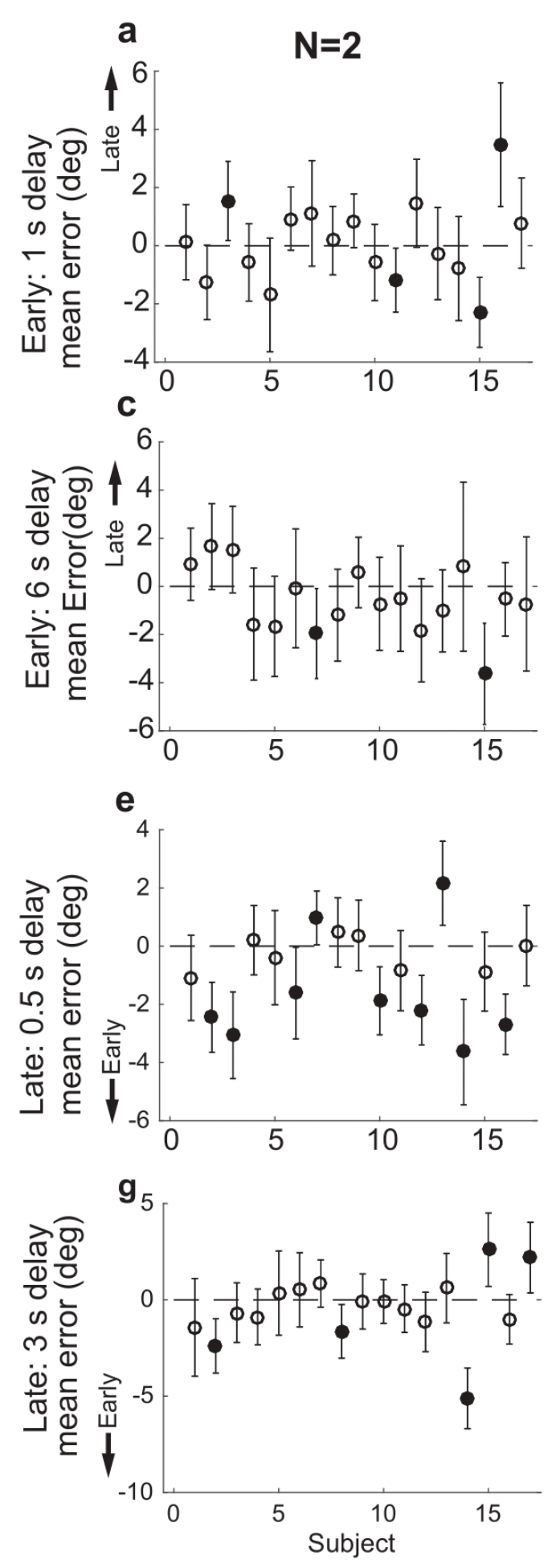
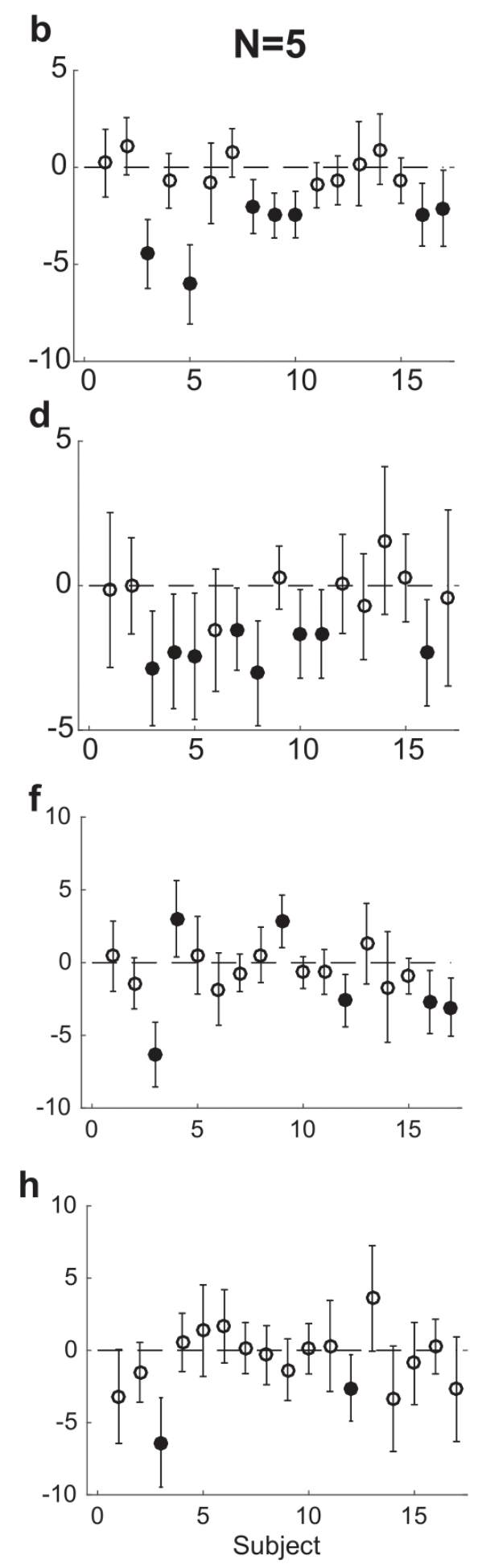

Supplemental Figure S5: Subject-wise mean error in the Sequential Perceived condition. a) Delay $=1 \mathrm{~s}$, set size $=2$ for Early samples. b) Delay $=1$, set size $=5$ for Early samples. c-d) as in $\mathbf{a}-\mathbf{b}$ but for delay of $6 \mathrm{~s}$. e) Delay $=0.5 \mathrm{~s}$, set size $=2$ for Late samples. f) Delay $=0.5 \mathrm{~s}$, set size $=5$ for Late samples. $\mathbf{g}-\mathbf{h})$ as in $\mathbf{e}-\mathbf{f}$ but for delay of $3 \mathrm{~s}$. In all panels, errorbars are $\pm 95 \%$ confidence intervals. Filled points indicate that 0 is not included in the confidence interval; i.e., there is bias in subject errors. 

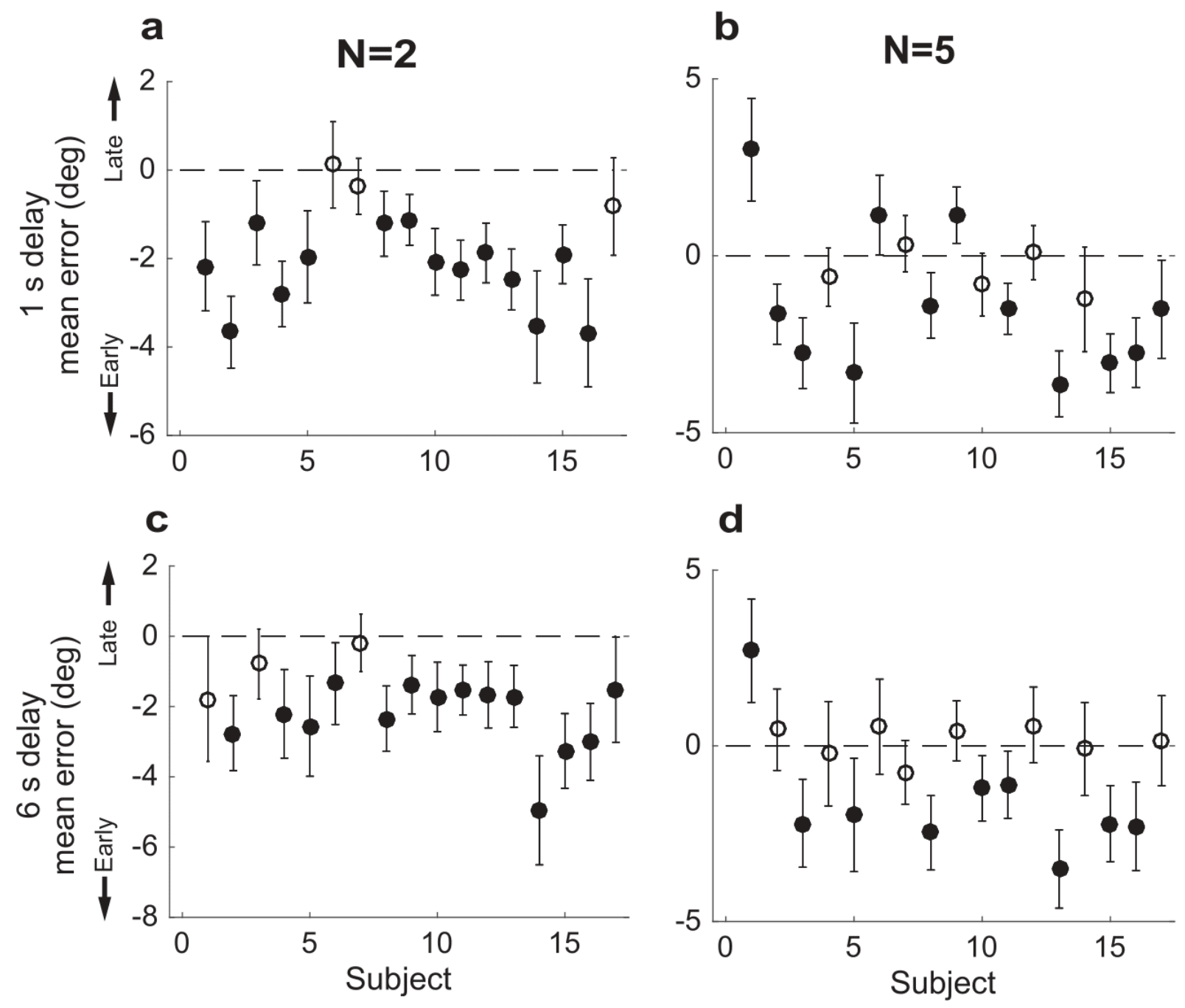

Supplemental Figure S6: Subject-wise mean error in the Sequential Computed condition. a) Delay $=1 \mathrm{~s}$, set $\operatorname{size}=2$. b) Delay $=1$, set $\operatorname{size}=5$. $\mathbf{c}-\mathbf{d}$ ) as in $\mathbf{a}-\mathbf{b}$ but for delay of $6 \mathrm{~s}$. In all panels, errorbars are $\pm 95 \%$ confidence intervals. Filled points indicate that 0 is not included in the confidence interval; i.e., there is bias in subject errors (which in this case tended to be towards the mean computed from the early points). 

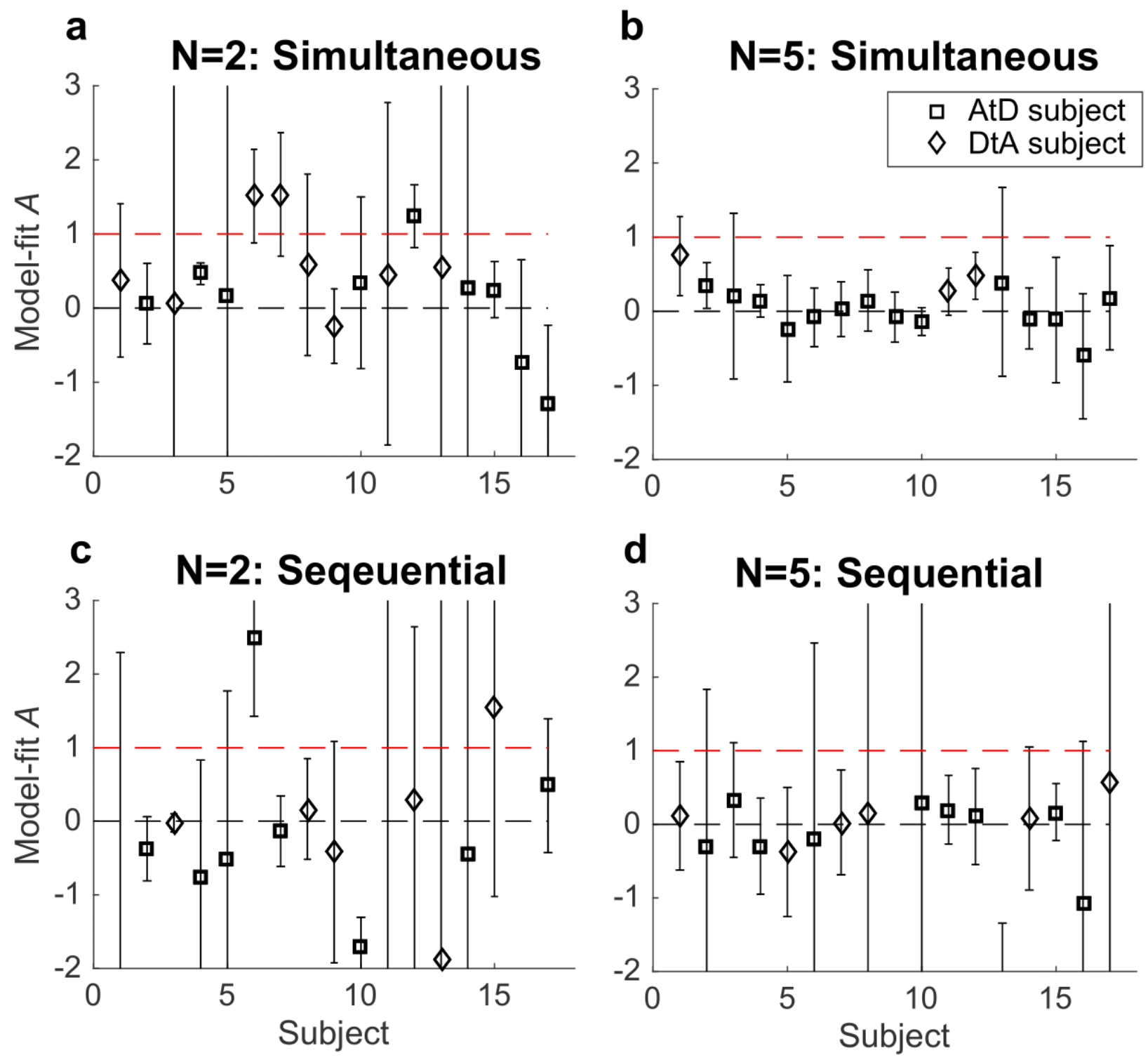

Supplemental Figure S7: Subject-based estimates of $A$. a,b) Simultaneous condition. a) Model-fits for $A$ for AtD (square) and DtA (diamond) participants at set size 2. b). Model-fits for $A$ for all participants at set size 5. c,d) Same as a,b but for the Sequential condition. In all panels, errorbars are $\pm 95 \%$ confidence intervals based on the Hessian computed during model fitting. Note that $A=0$ implies no difference between the diffusion constant for a single and $N$ points, whereas $A=1$ implies that the variance and diffusion constant relationship predictions of the AtD and DtA models are equal and thus the models cannot be distinguished from each other. 

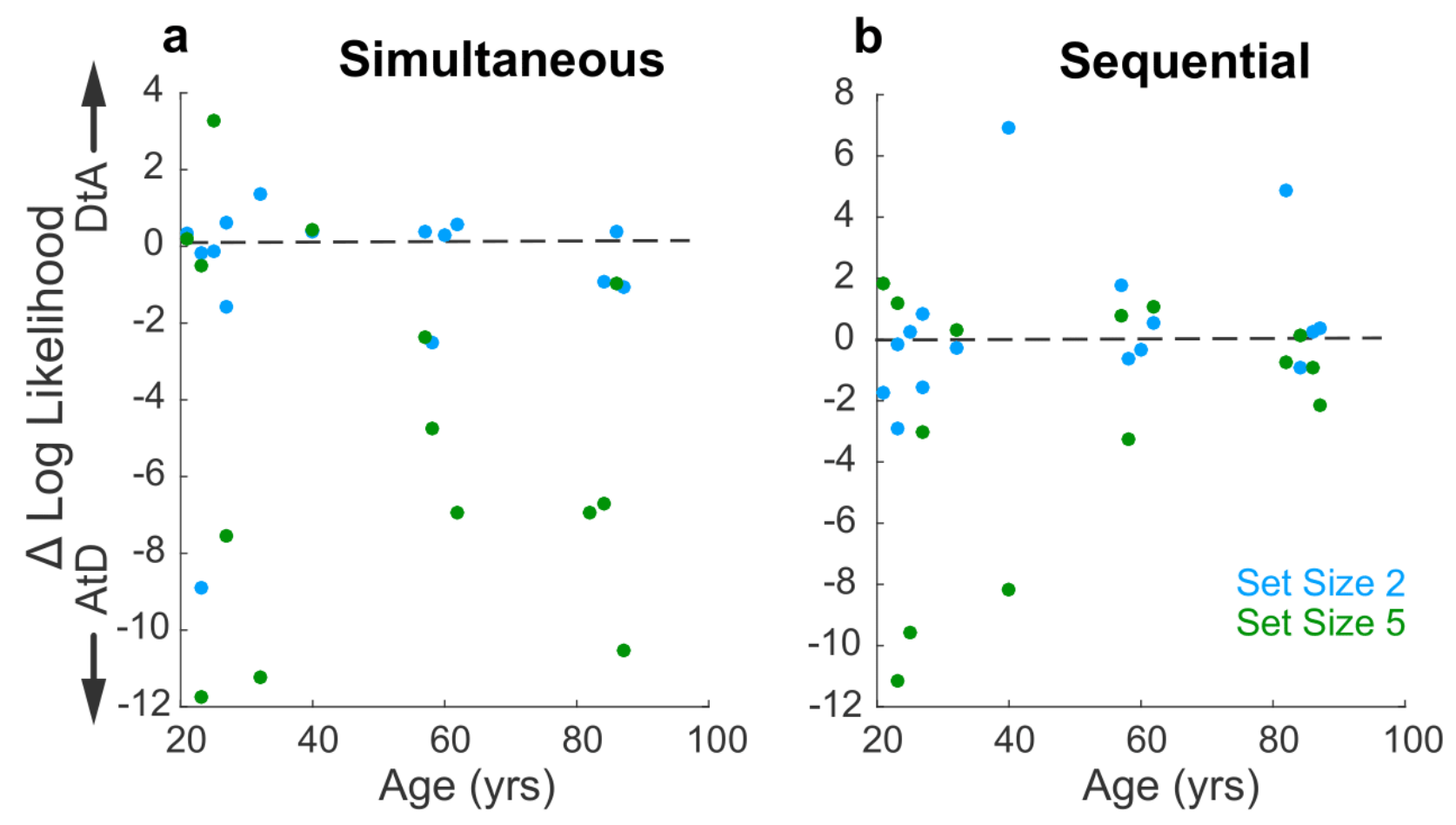

Supplemental Figure S8: Relationship between log likelihood difference for the two strategies and age. a) Log likelihood comparison for AtD and DtA (negative favors AtD) for set sizes 2 and 5 under Simultaneous conditions is not dependent upon age (correlation, $p s>0.20$ computed separately for each set size ). b) Same as in A, but for the Sequential Conditions ( $p s>0.20)$. 